\title{
On a Ferromagnetic Spin Chain
}

\author{
Andreas Knauf \\ Technische Universität, Fachbereich 3 - Mathematik, MA 7-2, Strasse des 17. Juni 135, \\ W-1000 Berlin 2, Germany
}

Received May 28, 1992; in revised form August 19, 1992

\begin{abstract}
The quotient $\zeta(s-1) / \zeta(s)$ of Riemann zeta functions is shown to be the partition function of a ferromagnetic spin chain for inverse temperature $s$.
\end{abstract}

\section{Contents}

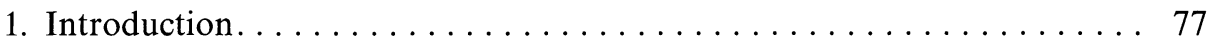

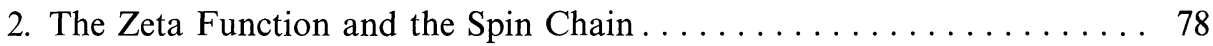

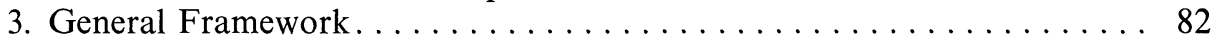

4. The Grand Canonical Ensemble ..................... 85

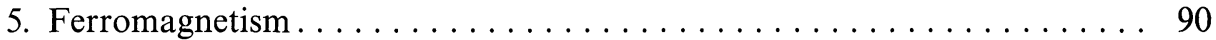

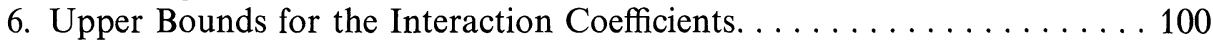

7. Asymptotic Translation Invariance . . . . . . . . . . . . . . . 103

8. Decay Properties of the Potential . . . . . . . . . . . . . . . . . . 104

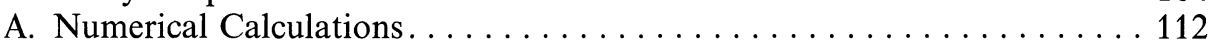

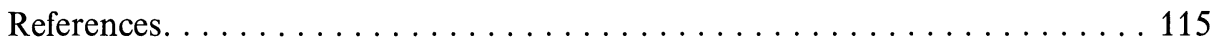

\section{Introduction}

The aim of this article is to relate ideas and concepts from statistical mechanics to the Riemann zeta function.

The quotient $Z(s)=\zeta(s-1) / \zeta(s)$ is interpreted as the partition function of an infinite ferromagnetic spin chain.

The existence of a connection between number theory and statistical mechanics has been conjectured by Kac (see his Comments in Pólya [4], pp. 424-426), Newman [3], Ruelle [5] and others.

One motivation for that conjecture has been the Lee-Yang circle theorem of statistical mechanics. In its basic form it states that all zeroes of the partition function of a ferromagnetic Ising model in the complex activity plane have unit modulus. 
Here we are interested in the zeroes of $Z(s)$ in the complex $s$ plane, $s$ being the inverse temperature. There exist variants of the Lee-Yang circle theorem which predict zero-free half-planes of the inverse temperature for certain ferromagnets, see, e.g., Ruelle [7]. Unfortunately, these theorems do not apply to our situation since our spin chain includes many-body interactions.

We begin in Sect. 2 by approximating $Z(s)$ by the partition functions $Z_{k}$ of chains of $k$ (classical) spins. This approximation is uniform for $\operatorname{Re}(s)>2+\varepsilon, \varepsilon>0$.

In Sect. 3 we introduce some notation. We decompose the canonical energy function $\mathbf{H}_{k}^{C}\left(\sigma_{1}, \ldots, \sigma_{k}\right), \sigma_{i} \in\{0,1\}$, in the form

$$
\mathbf{H}_{k}^{C}(\sigma)=-\sum_{t_{1} \in\{0,1\}} j_{k}^{C}\left(t_{1}, \ldots, t_{k}\right) \cdot(-1)^{\sigma \cdot t} .
$$

Proposition 3.2 states a necessary and sufficient condition for the preservation of the ferromagnetic property $j_{k}^{C}(t) \geqq 0$ under nonlinear transformations of the values of an energy function.

In order to obtain bounds on the canonical interaction coefficients $j_{k}^{C}(t)$, it turns out to be convenient to consider the grand canonical ensemble, too. This is done in Sect. 4.

There are no couplings to an external field. More general, the grand canonical interaction coefficients $j_{k}^{G}(t)$ between an odd number of spins vanish (Proposition 4.3). Furthermore, we have the mirror symmetry

$$
j_{k}^{G}\left(t_{1}, \ldots, t_{k}\right)=j_{k}^{G}\left(t_{k}, \ldots, t_{1}\right)
$$

(Proposition 4.9).

In Sect. 5 we show that the canonical and grand canonical ensembles are (weakly) ferromagnetic in the sense

$$
j_{k}^{C}(t) \geqq 0 \text { and } j_{k}^{G}(t) \geqq 0 \text { for } t \neq 0 .
$$

More precisely, we obtain inequalities between the interaction coefficients for spin chains of length $k$ and of length $k+1$. The proof uses interpolation techniques and Proposition 3.2.

In Sect. 6 upper bounds for the canonical interaction coefficients $j_{k}^{C}(t)$ are obtained.

The interaction coefficients are not translation invariant, but their variation under translations is small as long as no spins near the edges of the chain are involved. In Sect. 7 we prove this property of asymptotic translation invariance.

A thermodynamic limit can only exist if the interaction coefficients decay fast enough. On the other hand, it is known that one-dimensional spin chains have no phase transitions at positive (real) temperatures if the interaction is of finite range or decays too fast. In Sect. 8 we give estimates for the decay properties of our model.

In the Appendix we show the result of a numerical calculation of $\mathbf{H}_{k}^{C}(\sigma)$ and of the interaction coefficients.

\section{The Zeta Function and the Spin Chain}

Riemann's zeta function $\zeta(s)$ is defined for $\operatorname{Re}(s)>1$ by

$$
\zeta(s):=\sum_{n=1}^{\infty} n^{-s}
$$


and for arbitrary $s \in \mathbb{C}$ by analytic continuation.

Instead of $\zeta(s)$ we study the quotient

$$
Z(s):=\frac{\zeta(s-1)}{\zeta(s)} .
$$

We denote by $\varphi: \mathbb{N} \rightarrow \mathbb{N}$ the Euler totient function. So $\varphi(n)$ is defined to be the number of positive integers not exceeding $n$ which are relatively prime w.r.t. $n$. Then

$$
Z(s)=\sum_{n=1}^{\infty} \varphi(n) \cdot n^{-s},
$$

as follows from the multiplication theorem for Dirichlet series (see, e.g., Apostol [1], p. 229).

We shall approximate $Z$ by a sequence $Z_{k}$ of functions, with $k \in \mathbb{N}_{0}$. These functions have the form

$$
Z_{k}(s):=\sum_{n=1}^{\infty} \varphi_{k}(n) \cdot n^{-s},
$$

where the $\varphi_{k}: \mathbb{N} \rightarrow \mathbb{N}_{0}$ are approximants of $\varphi$.

For $k \in \mathbb{N}_{0}$ we define inductively the coefficients $h_{k}^{C}: G_{k} \rightarrow \mathbb{N}$ on the cyclic groups $G_{k}:=\mathbb{Z}_{2^{k}} \equiv \mathbb{Z} /\left(2^{k} \cdot \mathbb{Z}\right)$ of order $2^{k}$. Whenever needed, we represent the group $G_{k}$ using the complete residue system $\left\{0, \ldots, 2^{k}-1\right\}$. The coefficients $h_{k}^{C}$ are then given by setting $h_{0}^{C}(0):=1$ and for $a \in G_{k}$

$$
h_{k+1}^{C}(2 a):=h_{k}^{C}(a), \quad h_{k+1}^{C}(2 a+1):=h_{k}^{C}(a)+h_{k}^{C}(a+1),
$$

the map

$$
\left\{0, \ldots, 2^{k}-1\right\} \rightarrow\left\{0, \ldots, 2^{k+1}-1\right\}, \quad a \mapsto 2 a,
$$

inducing the monomorphism $G_{k} \rightarrow G_{k+1}$ used in (5).

As an example, we have for $k=3$ the values $h_{k}^{C}(0)=1, h_{k}^{C}( \pm 1)=4, h_{k}^{C}( \pm 2)$ $=3, h_{k}^{C}( \pm 3)=5$ and $h_{k}^{C}(4)=2$.

The index $C$ of $h_{k}^{C}$ symbolizes the canonical ensemble. Later on we will introduce the grand canonical ensemble. The objects connected with that ensemble will then carry a superscript $G$.

Then the $\varphi_{k}$ are given by

$$
\varphi_{k}(n):=\#\left\{a \in G_{k} \mid h_{k}^{C}(a)=n\right\}
$$

(so $\varphi_{3}(1)=\varphi_{3}(2)=1, \varphi_{3}(3)=\varphi_{3}(4)=\varphi_{3}(5)=2$, and $\varphi_{3}(k)=0$ for $k \geqq 6$ ).

We start by collecting some elementary properties of the $\varphi_{k}$. We call the base two logarithm

$$
L_{k}(a):=\log _{2}(\operatorname{Ord}(a))
$$

of the order of a group element $a \in G_{k}$ its $L$-order. Note that $L_{k}(a) \in\{0, \ldots, k\}$ since the order of an element (i.e., the order of the cyclic subgroup generated by that element) divides the order of the group.

Furthermore, we denote by $\mathrm{F}(k)$ the $k$-th Fibonacci number, that is,

$$
\mathrm{F}(1)=\mathrm{F}(2)=1, \quad \mathrm{~F}(k+2)=\mathrm{F}(k)+\mathrm{F}(k+1) .
$$


Lemma 2.1. For $k \in \mathbb{N}_{0}$ and $a \in G_{k}$

$$
h_{k}^{C}(-a)=h_{k}^{C}(a) \text {, }
$$

and

$$
L_{k}(a)+1 \leqq h_{k}^{C}(a) \leqq \mathrm{F}\left(L_{k}(a)+2\right) .
$$

For $k \in \mathbb{N}$ adjacent coefficients are relatively prime:

$$
\operatorname{gcd}\left(h_{k}^{c}(a), h_{k}^{c}(a+1)\right)=1 .
$$

Moreover, for every relatively prime pair $(q, p), 1 \leqq q<p \leqq k+1$, there is exactly one $l \leqq k$ and $a \in G_{l}$ with

$$
(q, p)=\left(h_{l}^{c}(a), h_{l}^{c}(a+1)\right) .
$$

Proof. The symmetry property (8) follows immediately from definition (5).

For $k=0$ the inequalities $(9)$ state that $h_{0}^{C}(0)=1$. We assume that they are true for $k$. Then all elements $a \in G_{k+1}$ with $L$-order $L_{k+1}(a) \leqq k$ are of the form $a=2 b$ with $b \in G_{k}$. For these $a$ we have

$$
L_{k+1}(a)+1=L_{k}(b)+1 \leqq h_{k}^{C}(b)=h_{k+1}^{c}(a) .
$$

If $L_{k+1}(a)=k+1$, then $L_{k+1}(a)+1=k+2 \leqq h_{k+1}^{C}(a)$, since then $h_{k+1}^{C}(a)$ is a sum of $k+2$ integers.

We perform the inductive step for the second inequality in (9) by assuming $h_{k}^{C}(b) \leqq \mathrm{F}\left(L_{k}(b)+2\right)$ for all $b \in G_{k}$. Then either $a \in G_{k+1}$ is of the form $a=2 b$, or $a$ is of maximal order, i.e. $L_{k+1}(a)=k+1$. In the first case we have

$$
h_{k+1}^{c}(a)=h_{k}^{c}(b) \leqq \mathrm{F}\left(L_{k}(b)+2\right)=\mathrm{F}\left(L_{k+1}(a)+2\right) .
$$

If $L_{k+1}(a)=k+1$, then for some $b \in G_{k}$

$$
h_{k+1}^{c}(a)=h_{k}^{C}(b)+h_{k}^{c}(b+1) \text {, }
$$

and either $L_{k}(b)=k, L_{k}(b+1)<k$ or conversely $L_{k}(b+1)=k, L_{k}(b)<k$, since only every second element in $G_{k}$ has $L$-order $k$. Then

$$
\begin{aligned}
h_{k+1}^{C}(a) & \leqq \mathrm{F}\left(L_{k}(b)+2\right)+\mathrm{F}\left(L_{k}(b+1)+2\right) \leqq \mathrm{F}(k+2)+\mathrm{F}(k+1) \\
& =\mathrm{F}\left(L_{k+1}(a)+2\right) .
\end{aligned}
$$

Adjacent elements are relatively prime if $k=1$, since then

$$
\operatorname{gcd}\left(h_{k}^{c}(a), h_{k}^{c}(a+1)\right)=\operatorname{gcd}(1,2)=1 .
$$

We assume (10) to hold for $k \in \mathbb{N}$. Then for $a \in G_{k+1}$ of the form $a=2 b$

$$
\begin{aligned}
\operatorname{gcd}\left(h_{k+1}^{c}(a), h_{k+1}^{c}(a+1)\right) & =\operatorname{gcd}\left(h_{k}^{C}(b), h_{k}^{C}(b)+h_{k}^{C}(b+1)\right) \\
& =\operatorname{gcd}\left(h_{k}^{C}(b), h_{k}^{C}(b+1)\right)=1,
\end{aligned}
$$

and similarly for $a=2 b+1$.

For $k=1$ the last assertion of the lemma holds since

$$
(1,2)=\left(h_{l}^{C}(a), h_{l}^{C}(a+1)\right)
$$

exactly if $l=k=1$ and $a=0$. 
Assume that we have shown that assertion up to order $k \in \mathbb{N}$. Then if $p \leqq k+1$ and $h_{k+1}^{C}(a)=p$, then by the first inequality in (9) $L_{k+1}(a) \leqq k$ so that we already treated that case. So we assume $p=k+2$. All relatively prime pairs $\left(q_{0}, p_{0}\right)$ with $q_{0}+p_{0}=p=k+2$ appear in the form $\left(h_{l}^{C}(b), h_{l}^{C}(b+1)\right)$ with $l \leqq k$. Then

$$
\left(h_{l+1}^{c}(2 b), h_{l+1}^{C}(2 b+1)\right)=\left(q_{0}, p\right)
$$

and

$$
\left(h_{l+1}^{C}(-2(b+1)), h_{l+1}^{C}(-2 b-1)\right)=\left(p_{0}, p\right) .
$$

So we find in our list all relatively prime pairs $(q, p), 1 \leqq q<p=k+2$ exactly once.

With the help of Lemma 2.1 we can describe how the $\varphi_{k}$ approximate Euler's totient:

Proposition 2.2. For $k \in \mathrm{N}_{0}$ we have

and

$$
\varphi_{k} \leqq \varphi_{k+1} \leqq \varphi,
$$

$$
\varphi_{k}(p)=\varphi(p) \text { for } 1 \leqq p \leqq k+1 .
$$

For $p>k+1, \varphi_{k}(p)<\varphi(p)$. For $k \geqq 2$ one has $\varphi_{k}(\mathrm{~F}(k+2))=2$ and

$$
\varphi_{k}(p)=0 \text { for } p>\mathrm{F}(k+2) \text {. }
$$

Proof. The inequality $\varphi_{k} \leqq \varphi_{k+1}$ in (12) follows immediately from the definitions (5) and (6). The second inequality is then a consequence of the first one and of assertion (13).

$\varphi_{0}(1)=1=\varphi(1)$, and $\varphi_{0}(p)=0<\varphi(p)$ for $p>1$, proving (13) for $k=0$.

To prove (13) for $k \in \mathbb{N}$, we fix $p \in\{1, \ldots, k+1\}$ and choose an arbitrary $q<p$ with $\operatorname{gcd}(q, p)=1$. We know from the last statement of Lemma 2.1 that there is exactly one $l \leqq k$ and $b \in G_{l}$ with $(q, p)=\left(h_{l}^{C}(b-1), h_{l}^{C}(b)\right)$. But $h_{l}^{C}(b)=h_{l+1}^{C}(2 b)=h_{k}^{C}\left(2^{k-l} b\right)$ so that $h_{k}^{C}(a)=p$ for $a=2^{k-l} b$. Moreover, for $q_{1} \neq q_{2}$ the corresponding $a_{1}, a_{2} \in G_{k}$ are different from each other (since the coefficient $h_{m}^{C}\left(2^{m-l} b-1\right)$ adjacent to $h_{m}^{C}\left(2^{m-l} b\right)$ is smaller than $h_{m}^{C}\left(2^{m-l} b\right)$ only if $m=l$ ).

Thus we have established a one-to-one correspondence between the $a \in G_{k}$ with $h_{k}^{C}(a)=p$ and the numbers $q<p$ relatively prime to $p$, proving (13).

Now we show that $\varphi_{k}(p)<\varphi(p)$ for $p \geqq k+2$. We have for $l \in \mathbb{N}$

$$
h_{l}^{C}( \pm 1)=h_{l-1}^{C}(0)+h_{l-1}^{C}( \pm 1)=1+h_{k-1}^{C}( \pm 1)
$$

and $h_{1}^{C}( \pm 1)=2$ so that $h_{l}^{C}( \pm 1)=l+1$. But this implies that $\varphi_{p-2}(p)$ $<\varphi_{p-1}(p)$. On the other hand, by $(12) \varphi_{p-1}(p) \leqq \varphi(p)$, proving $\varphi_{p-2}(p)<\varphi(p)$ and $\varphi_{k}(p)<\varphi(p)$ for $p \geqq k+2$.

Equation (14) follows from inequality (9) in Lemma 2.1.

To show that for $k \geqq 2$ one has $\varphi_{k}(\mathrm{~F}(k+2))=2$, we notice that for $k=2$ there are exactly two $a \in G_{2}$ with $h_{2}^{c}(a)=\mathrm{F}(2+2)=3$, namely $a=1$ and $a=-1$ $\equiv 3(\bmod 4)$. Notice that for both $a$ exactly one of the neighbours $a \pm 1$ has $h_{2}^{C}$-value $\mathrm{F}(2+1)=2$.

But if for $k$ there are exactly two $a \in G_{k}$ with $h_{k}^{C}(a)=\mathrm{F}(k+2)$, both $a$ having exactly one neighbour $a \pm 1$ with $h_{k}^{C}(a \pm 1)=\mathrm{F}(k+1)$, then a similar statement holds for $k+1$. 
Corollary 2.3. For all $\varepsilon>0$ the sequence $\left\{Z_{k}\right\}_{k \in \mathbb{N}_{0}}$ of functions converges uniformly to $Z$ in the half plane $\{s \in \mathbb{C} \mid \operatorname{Re}(s)>2+\varepsilon\}$.

Proof. For $\operatorname{Re}(s)>2+\varepsilon$,

$$
\begin{aligned}
\left|Z(s)-Z_{k}(s)\right| & \leqq \sum_{l=k+2}^{\infty} \varphi(l) \cdot l^{-(2+\varepsilon)} \\
& \leqq \sum_{l=k+2}^{\infty} l^{-(1+\varepsilon)}<\frac{1}{\varepsilon}(k+1)^{-\varepsilon},
\end{aligned}
$$

since $\varphi(n) \leqq n$.

Clearly, one could equally well approximate $Z(s)$ in its half plane $\operatorname{Re}(s)>2$ of convergence just by truncating $\varphi(n)$ after the $k$-th term. However the chosen form of the approximation exhibits an asymptotic (as $k \rightarrow \infty$ ) self-similarity which will be useful for the study of $Z(s)$ in the whole complex plane.

\section{General Framework}

We interpret the binary expansion $\sigma=\left(\sigma_{1}, \ldots, \sigma_{k}\right) \in \mathbf{G}_{k}:=\left(\mathbb{Z}_{2}\right)^{k}$ of an element $a \in G_{k}$ as a configuration of a spin chain with $k$ spins $\sigma_{i} \in \mathbb{Z}_{2} \equiv \mathbb{Z} / 2 \mathbb{Z}$, the $i^{\text {th }}$ spin being in a downward (resp. upward) position for $\sigma_{i}=0$ (resp. 1). More precisely, using the complete residue systems $\left\{0, \ldots, 2^{k}-1\right\}$ for $G_{k}$ and $\{0,1\}$ for $\mathbb{Z}_{2}$, respectively, we represent $a \in G_{k}$ uniquely in the form

$$
a=\sum_{i=1}^{k} \sigma_{i} 2^{k-i}, \quad \sigma_{i} \in \mathbb{Z}_{2} .
$$

This prescription defines for $k \in \mathbb{N}_{0}$ set theoretical bijections

$$
\mathrm{Id}_{k}: G_{k} \rightarrow \mathbf{G}_{k}, \quad a \mapsto\left(\sigma_{1}, \ldots, \sigma_{k}\right) .
$$

We will use the functions

$$
\mathbf{h}_{k}^{C}:=h_{k}^{C} \circ \operatorname{Id}_{k}^{-1} \quad \text { and } \quad \mathbf{H}_{k}^{C}:=H_{k}^{C} \circ \operatorname{Id}_{k}^{-1}
$$

on $\mathbf{G}_{k}$, with $H_{k}^{C}:=\ln h_{k}^{C}$.

Lemma 3.1. For $a \in G_{k}$ let $\sigma=\left(\sigma_{1}, \ldots, \sigma_{k}\right):=\operatorname{Id}_{k}(a)$. Then

$$
\operatorname{Id}_{k}(-(a+1))=\left(1-\sigma_{1}, \ldots, 1-\sigma_{k}\right) .
$$

Proof. Modulo $2^{k}$ we have

$$
-a-1=-\sum_{i=1}^{k} \sigma_{i} 2^{k-i}-\left(2^{k}-\sum_{i=1}^{k} 2^{k-i}\right)=\sum_{i=1}^{k}\left(1-\sigma_{i}\right) 2^{k-i} .
$$

Thus it follows from the definition (5) of $h_{k}^{C}$ that the functions $\mathbf{h}_{k}^{C}: \mathbf{G}_{k} \rightarrow \mathbb{N}$ are given by $\mathbf{h}_{0}^{C}=1$,

$$
\mathbf{h}_{k+1}^{C}(\sigma, 0)=\mathbf{h}_{k}^{C}(\sigma) \text { and } \mathbf{h}_{k+1}^{C}(\sigma, 1)=\mathbf{h}_{k}^{C}(\sigma)+\mathbf{h}_{k}^{C}(1-\sigma),
$$

since by $(8) \mathbf{h}_{k}^{C}(1-\sigma)=h_{k}^{C}(-(a+1))=h_{k}^{C}(a+1)$ for $\sigma=\left(\sigma_{1}, \ldots, \sigma_{k}\right)=\operatorname{Id}_{k}(a)$ and $1-\sigma:=\left(1-\sigma_{1}, \ldots, 1-\sigma_{k}\right)$. 
The group $\mathbf{G}_{k}^{*}$ of characters on $\mathbf{G}_{k}$ is isomorphic to $\mathbf{G}_{k}$, and for $t=\left(t_{1}, \ldots, t_{k}\right)$ $\in \mathbf{G}_{k}^{*}, k \in \mathbb{N}$, we write $\chi_{t}: \mathbf{G}_{k} \rightarrow\{+1,-1\}$ for the character function given by

$$
\chi_{t}(\sigma):=(-1)^{\sum_{i=1}^{k} \sigma_{i} \cdot t_{l}}, \quad \sigma \in \mathbf{G}_{k} .
$$

For $k=0$ we set $\chi_{0}:=+1$.

The set

$$
\mathscr{A}_{k}:=\left\{f: \mathbf{G}_{k} \rightarrow \mathbb{R}\right\}
$$

of real-valued observables, together with addition and multiplication, forms an algebra. We denote by $\mathscr{A}_{k}^{*}:=\left\{f: \mathbf{G}_{k}^{*} \rightarrow \mathbb{R}\right\}$ the algebra over the dual group.

Fourier transformation

$$
\mathscr{F}_{k}: \mathscr{A}_{k} \rightarrow \mathscr{A}_{k}^{*}, \quad f \mapsto \hat{f}
$$

is defined by

$$
\hat{f}(t):=2^{-k} \sum_{\sigma \in \mathbf{G}_{k}} f(\sigma) \chi_{t}(\sigma), \quad t \in \mathbf{G}_{k}^{*} .
$$

Its inverse $\mathscr{F}_{k}^{-1}: \mathscr{A}_{k}^{*} \rightarrow \mathscr{A}_{k}, g \mapsto \check{g}$, is then given by

$$
\check{g}(\sigma)=\sum_{t \in \mathbf{G}_{k}^{*}} g(t) \chi_{t}(\sigma), \quad \sigma \in \mathbf{G}_{k} .
$$

The multiplicative factor $2^{k}=\operatorname{Ord}\left(\mathbf{G}_{k}\right)$ appears in the orthogonality relation

$$
\sum_{\sigma \in \mathbf{G}_{k}} \chi_{t^{I}}(\sigma) \chi_{t^{I I}}(\sigma)= \begin{cases}2^{k} & \text { if } t^{I}=t^{I I} \\ 0 & \text { if } t^{I} \neq t^{I I}\end{cases}
$$

for the characters $t^{I}, t^{I I} \in \mathbf{G}_{k}^{*}$.

The most important observables are the canonical energy functions $\mathbf{H}_{k}^{C} \in \mathscr{A}_{k}$, $k \in \mathbb{N}_{0}$, since

$$
\begin{aligned}
Z_{k}(s) & =\sum_{n=1}^{\infty} \varphi_{k}(n) \cdot n^{-s}=\sum_{\sigma \in \mathbf{G}_{k}}\left(\mathbf{h}_{k}^{C}(\sigma)\right)^{-s} \\
& =\sum_{\sigma \in \mathbf{G}_{k}} \exp \left(-s \cdot \mathbf{H}_{k}^{C}(\sigma)\right) .
\end{aligned}
$$

We call the values

$$
j_{k}^{C}(t):=-\mathscr{F}_{k}\left(\mathbf{H}_{k}^{C}\right)(t), \quad t \in \mathbf{G}_{k}^{*},
$$

the canonical interaction coefficients.

An observable $f \in \mathscr{A}_{k}$ is called strictly ferromagnetic if $\hat{f} \geqq 0$ and (weakly) ferromagnetic if $\hat{f}(t) \geqq 0$ for $t \in \mathbf{G}_{k}^{*} \backslash\{0\}$. Clearly $-\mathbf{H}_{k}^{C}$ cannot be strictly ferromagnetic for $k \in \mathbb{N}$ since its mean value $j_{k}^{c}(0)<0$.

The strictly ferromagnetic observables form a multiplicative cone $\mathscr{C}_{k} \subset \mathscr{A}_{k}$, that is, for $f, g \in \mathscr{C}_{k}$ and $\lambda \geqq 0$

$$
\lambda f \in \mathscr{C}_{k}, \quad f+g \in \mathscr{C}_{k} \text { and } f \cdot g \in \mathscr{C}_{k},
$$

see Ginibre [2]. The last statement holds true since Fourier transformation changes ordinary product into convolution product. 
The following observation will turn out to be helpful:

Proposition 3.2. For $y \in \mathbb{R}^{+} \cup\{\infty\}$ let $g \in C^{\infty}((-y, y)$, $\mathbb{R})$ be a function whose expansion

$$
g(x)=\sum_{i=0}^{\infty} c_{i} x^{i}
$$

is absolutely convergent on the interval $(-y, y)$. Consider the set

$$
Y_{k}:=\left\{f \in \mathscr{A}_{k} \mid f\left(\mathbf{G}_{k}\right) \subset(-y, y)\right\}
$$

of observables.

Then the maps $\mathscr{G}_{k}: Y_{k} \rightarrow \mathscr{A}_{k}, f \mapsto g \circ f$, preserve strict ferromagnetism, i.e.

$$
\mathscr{G}_{k}\left(\mathscr{C}_{k} \cap Y_{k}\right) \subset \mathscr{C}_{k} \quad \forall k \in \mathbb{N}_{0},
$$

if and only if $c_{i} \geqq 0$ for all $i \in \mathbb{N}_{0}$.

Proof. We assume that $c_{i} \geqq 0$ for all $i \in \mathbb{N}_{0}, f \in \mathscr{C}_{k} \cap Y_{k}$ and set $h:=\mathscr{G}_{k}(f)$. Then

$$
\hat{h}(t)=2^{-k} \sum_{\sigma \in \mathbf{G}_{k}} g(f(\sigma)) \chi_{t}(\sigma)=2^{-k} \sum_{\sigma \in \mathbf{G}_{k}}\left(\sum_{i=0}^{\infty} c_{i}(f(\sigma))^{i}\right) \chi_{t}(\sigma) .
$$

In order to show that $\hat{h} \geqq 0$, by linearity of the Fourier transformation it is sufficient to show strict ferromagnetism for the functions $f^{i}, i \in \mathbb{N}_{0}$. But by assumption $f \in \mathscr{C}_{k}$, and $\mathscr{C}_{k}$ is a multiplicative cone.

Conversely, assume that there is a $l \in \mathbb{N}_{0}$ with $c_{l}<0$. Then set $k:=l$ and for $\varepsilon>0$ let the Fourier transform $\hat{f}_{\varepsilon} \in \mathscr{A}_{k}^{*}$ of $f_{\varepsilon}$ be given by $\hat{f}_{\varepsilon}(t):=\varepsilon$ if $\#\{i \in\{1, \ldots, k\} \mid$ $\left.t_{i}=1\right\}=1$ and $\hat{f}_{\varepsilon}(t):=0$ otherwise. Then for $t:=(1, \ldots, 1) \in \mathbf{G}_{k}^{*}$

$$
\begin{aligned}
\mathscr{G}_{k}\left(f_{\varepsilon}\right)(t) & =\sum_{i=0}^{\infty} c_{i}\left(\sum_{\sigma \in \mathbf{G}_{k}}\left(\sum_{t^{\prime} \in \mathbf{G}_{k}^{*}} \hat{f}_{\varepsilon}\left(t^{\prime}\right) \chi_{t^{\prime}}(\sigma)\right)^{i} \chi_{t}(\sigma)\right) \\
& =2^{k} \sum_{i=0}^{\infty} c_{i}\left(\sum_{\substack{t_{1}, \ldots, t_{i} \in \mathbf{G}_{k}^{*} \\
t_{1}+. .+t_{i}=t}} \hat{f}_{\varepsilon}\left(t_{1}\right) \cdot \cdots \cdot \hat{f}_{\varepsilon}\left(t_{i}\right)\right) \\
& =2^{k} c_{k} \varepsilon^{k} k !+\mathcal{O}\left(\varepsilon^{k+2}\right)<0 \text { for } \varepsilon>0 \text { small. }
\end{aligned}
$$

Using this proposition we will show in Sect. 5 that $-\mathbf{H}_{k}^{C}$ is weakly ferromagnetic.

In Sect. 6 we will derive upper bounds for the canonical interaction coefficients $j_{k}^{C}(t)$. It will turn out, roughly speaking, that $j_{k}^{C}(t)$ is typically small if $\#_{k}(t)$ is odd,

$$
\#_{k}(t):=\#\left\{i \in\{1, \ldots, k\} \mid t_{i}=1\right\} \text { for } t \in \mathbf{G}_{k}^{*}
$$

denoting the counting function on the group $\mathbf{G}_{k}^{*}$ of characters.

For $t \in \mathbf{G}_{k}^{*} \backslash\{0\}$ we introduce the size

$$
S_{k}(t):=P_{k}^{r}(t)-P_{k}^{l}(t)+1
$$

of $t$, where

$$
P_{k}^{r}(t):=\max \left\{i \in\{1, \ldots, k\} \mid t_{i}=1\right\}
$$

and

$$
P_{k}^{l}(t):=\min \left\{i \in\{1, \ldots, k\} \mid t_{i}=1\right\} .
$$

For $\#_{k}(t)$ even, $j_{k}^{C}(t)$ is small only if the size of $t$ is large. 
In Sect. 7 we begin the study of the thermodynamic limit $k \rightarrow \infty$ by relating spin chains for different lengths $k$. To do this, we introduce some notation. The concatenation $\left(\sigma^{I}, \sigma^{I I}\right) \in \mathbf{G}_{k+l}$ of two spin configurations $\sigma^{I}=\left(\sigma_{1}^{I}, \ldots, \sigma_{k}^{I}\right) \in \mathbf{G}_{k}$ and $\sigma^{I I}=\left(\sigma_{1}^{I I}, \ldots, \sigma_{l}^{I I}\right) \in \mathbf{G}_{l}$ is given by

$$
\left(\sigma^{I}, \sigma^{I I}\right):=\left(\sigma_{1}^{I}, \ldots, \sigma_{k}^{I}, \sigma_{1}^{I I}, \ldots, \sigma_{l}^{I I}\right),
$$

and we omit brackets if appropriate.

For $\sigma=\left(\sigma_{1}, \ldots, \sigma_{k}\right) \in \mathbf{G}_{k}$ we write $1-\sigma \equiv 1+\sigma:=\left(1-\sigma_{1}, \ldots, 1-\sigma_{k}\right)$ $\in \mathbf{G}_{k}$ for the group element of the inverted spin configuration.

We introduce similar notations for the elements of the dual group $\mathbf{G}_{k}^{*}$ and observe that the character functions are multiplicative w.r.t. concatenation, that is, for $\sigma^{I} \in \mathbf{G}_{k}, \sigma^{I I} \in \mathbf{G}_{l}$ and $t^{I} \in \mathbf{G}_{k}^{*}, t^{I I} \in \mathbf{G}_{l}^{*}$

$$
\chi_{\left(t^{I}, t I I\right)}\left(\sigma^{I}, \sigma^{I I}\right)=\chi_{t^{I}}\left(\sigma^{I}\right) \cdot \chi_{t^{I I}}\left(\sigma^{I I}\right) .
$$

\section{The Grand Canonical Ensemble}

In this section we begin the study of the interaction coefficients.

Lemma 4.1. For $k \in \mathbb{N}_{0}$ and $t \in \mathbf{G}_{k}^{*}$ one has

$$
j_{k+1}^{C}(t, 0)+j_{k+1}^{C}(t, 1)=j_{k}^{C}(t)
$$

and if $\#_{k}(t)$ is odd, then

$$
j_{k+1}^{c}(t, 0)=j_{k+1}^{c}(t, 1)=\frac{1}{2} j_{k}^{C}(t)
$$

Proof. By (19),

$$
\begin{aligned}
j_{k+1}^{C}(t, 0)+j_{k+1}^{C}(t, 1) & =-2^{-(k+1)} \sum_{\sigma \in \mathbf{G}_{k}} \sum_{\tau \in \mathbf{G}_{1}} \mathbf{H}_{k+1}^{C}(\sigma, \tau) \chi_{t}(\sigma) \cdot\left(\chi_{0}(\tau)+\chi_{1}(\tau)\right) \\
= & -2^{-k} \sum_{\sigma \in \mathbf{G}_{k}} \mathbf{H}_{k+1}^{C}(\sigma, 0) \chi_{t}(\sigma) \\
= & -2^{-k} \sum_{\sigma \in \mathbf{G}_{k}} \mathbf{H}_{k}^{C}(\sigma) \chi_{t}(\sigma)=j_{k}^{C}(t),
\end{aligned}
$$

proving (20).

To derive (21), we note that for $\#_{k}(t)$ odd, $\chi_{t}(1-\sigma)=-\chi_{t}(\sigma)$ so that

$$
\begin{aligned}
j_{k+1}^{C}(t, 0)-j_{k+1}^{C}(t, 1) & =-2^{-(k+1)} \sum_{\sigma \in \mathbf{G}_{k}} \sum_{\tau \in \mathbf{G}_{1}} \mathbf{H}_{k+1}^{C}(\sigma, \tau) \chi_{t}(\sigma) \cdot\left(\chi_{0}(\tau)-\chi_{1}(\tau)\right) \\
& =-2^{-k} \sum_{\sigma \in \mathbf{G}_{k}} \mathbf{H}_{k+1}^{C}(\sigma, 1) \chi_{t}(\sigma) \\
& =-2^{-k} \sum_{\sigma \in \mathbf{G}_{k}}\left(\mathbf{H}_{k}^{C}(\sigma)+\mathbf{H}_{k}^{C}(1-\sigma)\right) \chi_{t}(\sigma) \\
& =-2^{-k} \sum_{\sigma \in \mathbf{G}_{k}}\left(\mathbf{H}_{k}^{C}(\sigma) \chi_{t}(\sigma)-\mathbf{H}_{k}^{C}(1-\sigma) \chi_{t}(1-\sigma)\right)=0,
\end{aligned}
$$

proving the first equation in (21).

The second equality in (21) follows from the first one and from (20). 
If $\#_{k}(t)$ is even, then we will see that $j_{k+1}^{C}(t, 0) \neq j_{k+1}^{C}(t, 1)$, and the difference between the two coefficients will be interpreted as the interaction coefficient $j_{k}^{G}(t)$ of a grand canonical ensemble. In the language of statistical mechanics this is an ensemble with a varying number of particles. Here the $L$-order of an element, introduced in (7), plays the role of a particle number.

For $s, z \in \mathbb{C}$ with $\operatorname{Re}(s)>2$ and $|z| \leqq 1$ the grand canonical ensemble

$$
\Xi(s, z):=1+\sum_{k=1}^{\infty} z^{k} \sum_{\sigma \in \mathbf{G}_{k-1}} \exp \left(-s \cdot \mathbf{H}_{k-1}^{G}(\sigma)\right)
$$

with

$$
\mathbf{h}_{k}^{G}: \mathbf{G}_{k} \rightarrow \mathbb{N}, \quad \mathbf{h}_{k}^{G}(\sigma):=\mathbf{h}_{k+1}^{C}(\sigma, 1) \quad \text { and } \quad \mathbf{H}_{k}^{G}:=\ln \left(\mathbf{h}_{k}^{G}\right)
$$

for $k \in \mathbb{N}_{0}$ is well-defined, and

$$
Z(s)=\Xi(s, 1)
$$

Exactly those elements $b \in G_{k+1}$ which are of the form $\operatorname{Id}_{k+1}(b)=(\sigma, 1)$ have $L$-order $k+1$.

Similar to the above treatment of the canonical ensemble we define for $k \in \mathbb{N}_{0}$ the grand canonical interaction coefficients

$$
j_{k}^{G}(t):=-\mathscr{F}_{k}\left(\mathbf{H}_{k}^{G}\right)(t) \text { for } t \in \mathbf{G}_{k}^{*} .
$$

Thus

$$
\mathbf{H}_{k}^{G}(\sigma)=-\sum_{t \in \mathbf{G}_{k}^{*}} j_{k}^{G}(t) \cdot \chi_{t}(\sigma), \quad \sigma \in \mathbf{G}_{k}
$$

The grand canonical and the canonical interaction coefficients are related as follows.

Lemma 4.2. For $k \in \mathbb{N}_{0}$ and $t \in \mathbf{G}_{k}^{*}$

$$
j_{k}^{G}(t)=2 j_{k+1}^{C}(t, 0)-j_{k}^{C}(t)=-2 j_{k+1}^{C}(t, 1)+j_{k}^{C}(t) .
$$

Proof.

$$
\begin{aligned}
2 j_{k+1}^{C}(t, 0)-j_{k}^{C}(t) & =-2^{-k} \sum_{\sigma \in \mathbf{G}_{k}}\left(\mathbf{H}_{k+1}^{C}(\sigma, 0)+\mathbf{H}_{k+1}^{C}(\sigma, 1)-\mathbf{H}_{k}^{C}(\sigma)\right) \chi_{t}(\sigma) \\
& =-2^{-k} \sum_{\sigma \in \mathbf{G}_{k}} \mathbf{H}_{k+1}^{C}(\sigma, 1) \chi_{t}(\sigma) \\
& =-2^{-k} \sum_{\sigma \in \mathbf{G}_{k}} \mathbf{H}_{k}^{G}(\sigma) \chi_{t}(\sigma)=j_{k}^{G}(t) .
\end{aligned}
$$

The second equation follows from (20).

The grand canonical interaction coefficients have two symmetries which are not shared by their canonical counterparts. 
Proposition 4.3. If $\#_{k}(t)$ is odd for $t \in \mathbf{G}_{k}^{*}$, then $j_{k}^{G}(t)=0$.

Proof. We have

$$
\mathbf{H}_{k}^{G}(1-\sigma)=\mathbf{H}_{k+1}^{C}(1-\sigma, 1)=\mathbf{H}_{k}^{C}(1-\sigma)+\mathbf{H}_{k}^{C}(\sigma)=\mathbf{H}_{k+1}^{C}(\sigma, 1)=\mathbf{H}_{k}^{G}(\sigma) .
$$

On the other hand, for $\#_{k}(t)$ odd

$$
\chi_{t}(1-\sigma)=(-1)^{\#_{k}(t)} \chi_{t}(\sigma)=-\chi_{t}(\sigma) .
$$

Thus

$$
\begin{aligned}
j_{k}^{G}(t) & =-2^{-k} \sum_{\sigma \in \mathbf{G}_{k}} \mathbf{H}_{k}^{G}(\sigma) \chi_{t}(\sigma) \\
& =+2^{-k} \sum_{\sigma \in \mathbf{G}_{k}} \mathbf{H}_{k}^{G}(1-\sigma) \chi_{t}(1-\sigma)=-j_{k}^{G}(t),
\end{aligned}
$$

proving the proposition.

The second symmetry property of the grand canonical interaction coefficients is a bit more subtle.

In order to gain more flexibility, we introduce for $k \in \mathbb{N}$ and $s_{0}, s_{1} \in \mathbb{R}$ the auxiliary functions $\mathbf{r}_{k}\left(s_{0}, s_{1}\right): \mathbf{G}_{k} \rightarrow \mathbb{R}$ by setting

$$
\mathbf{r}_{1}\left(s_{0}, s_{1}\right)(0):=s_{0}, \quad \mathbf{r}_{1}\left(s_{0}, s_{1}\right)(1):=s_{1}
$$

and for $\sigma \in \mathbf{G}_{k}$

$$
\mathbf{r}_{k+1}\left(s_{0}, s_{1}\right)(\sigma, 0):=\mathbf{r}_{k}\left(s_{0}, s_{1}\right)(\sigma)
$$

and

$$
\mathbf{r}_{k+1}\left(s_{0}, s_{1}\right)(\sigma, 1):=\mathbf{r}_{k}\left(s_{0}, s_{1}\right)(\sigma)+\mathbf{r}_{k}\left(s_{0}, s_{1}\right)(1-\sigma) .
$$

In particular we have $\mathbf{h}_{k}^{C}=\mathbf{r}_{k}(1,2)$ for $k \in \mathbb{N}$.

Lemma 4.4. For $k \in \mathbb{N}$ and $s_{0}, s_{1} \in \mathbb{R}$ we have

$$
\mathbf{r}_{k}\left(s_{0}, s_{1}\right)=s_{0} \cdot \mathbf{r}_{k}(1,0)+s_{1} \cdot \mathbf{r}_{k}(0,1) .
$$

Proof. For $k=1, \mathbf{r}_{1}\left(s_{0}, s_{1}\right)(\sigma)=s_{\sigma}$. Assuming (25) for $k \in \mathbb{N}$, we have

$$
\begin{aligned}
\mathbf{r}_{k+1}\left(s_{0}, s_{1}\right)(\sigma, 0) & =\mathbf{r}_{k}\left(s_{0}, s_{1}\right)(\sigma)=s_{0} \cdot \mathbf{r}_{k}(1,0)(\sigma)+s_{1} \cdot \mathbf{r}_{k}(0,1)(\sigma) \\
& =s_{0} \cdot \mathbf{r}_{k+1}(1,0)(\sigma, 0)+s_{1} \cdot \mathbf{r}_{k+1}(0,1)(\sigma, 0)
\end{aligned}
$$

and

$$
\begin{aligned}
\mathbf{r}_{k+1}\left(s_{0}, s_{1}\right)(\sigma, 1)= & \mathbf{r}_{k}\left(s_{0}, s_{1}\right)(\sigma)+\mathbf{r}_{k}\left(s_{0}, s_{1}\right)(1-\sigma) \\
= & s_{0} \mathbf{r}_{k}(1,0)(\sigma)+s_{1} \mathbf{r}_{k}(0,1)(\sigma)+s_{0} \mathbf{r}_{k}(1,0)(1-\sigma) \\
& +s_{1} \mathbf{r}_{k}(0,1)(1-\sigma) \\
= & s_{0} \cdot \mathbf{r}_{k+1}(1,0)(\sigma, 1)+s_{1} \cdot \mathbf{r}_{k+1}(0,1)(\sigma, 1) . \quad \square
\end{aligned}
$$

Lemma 4.5. For $k \in \mathbb{N}, s_{0}, s_{1} \in \mathbb{R}$ and $\left(\sigma_{1}, \ldots, \sigma_{k}\right) \in \mathbf{G}_{k}$ we have

$$
\mathbf{r}_{k}\left(s_{1}, s_{0}\right)\left(\sigma_{1}, \sigma_{2}, \ldots, \sigma_{k}\right)=\mathbf{r}_{k}\left(s_{0}, s_{1}\right)\left(1-\sigma_{1}, \sigma_{2}, \ldots, \sigma_{k}\right) .
$$


Proof. For $k=1, \mathbf{r}_{1}\left(s_{1}, s_{0}\right)(\sigma)=s_{1-\sigma}=\mathbf{r}_{1}\left(s_{0}, s_{1}\right)(1-\sigma)$. Assuming (26) for $k \in \mathbb{N}$, we have

$$
\begin{aligned}
\mathbf{r}_{k+1}\left(s_{1}, s_{0}\right)\left(\sigma_{1} \ldots, \sigma_{k}, 0\right) & =\mathbf{r}_{k}\left(s_{1}, s_{0}\right)\left(\sigma_{1}, \ldots, \sigma_{k}\right) \\
& =\mathbf{r}_{k}\left(s_{0}, s_{1}\right)\left(1-\sigma_{1}, \sigma_{2}, \ldots, \sigma_{k}\right) \\
& =\mathbf{r}_{k+1}\left(s_{0}, s_{1}\right)\left(1-\sigma_{1}, \sigma_{2}, \ldots, \sigma_{k}, 0\right)
\end{aligned}
$$

and

$$
\begin{aligned}
\mathbf{r}_{k+1}\left(s_{1}, s_{0}\right)\left(\sigma_{1} \ldots, \sigma_{k}, 1\right)= & \mathbf{r}_{k}\left(s_{1}, s_{0}\right)\left(\sigma_{1}, \ldots, \sigma_{k}\right)+\mathbf{r}_{k}\left(s_{1}, s_{0}\right)\left(1-\sigma_{1}, \ldots, 1-\sigma_{k}\right) \\
= & \mathbf{r}_{k}\left(s_{0}, s_{1}\right)\left(1-\sigma_{1}, \sigma_{2}, \ldots, \sigma_{k}\right) \\
& +\mathbf{r}_{k}\left(s_{0}, s_{1}\right)\left(\sigma_{1}, 1-\sigma_{2}, \ldots, 1-\sigma_{k}\right) \\
= & \mathbf{r}_{k+1}\left(s_{0}, s_{1}\right)\left(1-\sigma_{1}, \sigma_{2}, \ldots, \sigma_{k}, 1\right) .
\end{aligned}
$$

Lemma 4.6. For $k \in \mathbb{N}, \sigma \in \mathbf{G}_{k}, l \in \mathbb{N}_{0}, \tau \in \mathbf{G}_{l}$ and $s_{0}, s_{1} \in \mathbb{R}$

$$
\mathbf{r}_{k+l}\left(s_{0}, s_{1}\right)(\sigma, \tau)=\mathbf{r}_{l+1}\left(\mathbf{r}_{k}\left(s_{0}, s_{1}\right)(\sigma), \mathbf{r}_{k}\left(s_{0}, s_{1}\right)(1-\sigma)\right)(0, \tau)
$$

Proof. The relation is shown by induction in $l \in \mathbb{N}_{0}$.

For $l=0(\sigma, \tau)=\sigma$ so that

$$
\begin{aligned}
\mathbf{r}_{k+l}\left(s_{0}, s_{1}\right)(\sigma, \tau) & =\mathbf{r}_{k}\left(s_{0}, s_{1}\right)(\sigma) \\
& =\mathbf{r}_{1}\left(\mathbf{r}_{k}\left(s_{0}, s_{1}\right)(\sigma), \mathbf{r}_{k}\left(s_{0}, s_{1}\right)(1-\sigma)\right)(0),
\end{aligned}
$$

since $\mathbf{r}_{1}\left(x_{0}, x_{1}\right)(0)=x_{0}$.

Setting $x_{0}:=\mathbf{r}_{k}\left(s_{0}, s_{1}\right)(\sigma)$ and $x_{1}:=\mathbf{r}_{k}\left(s_{0}, s_{1}\right)(1-\sigma)$, we assume that

$$
\mathbf{r}_{k+l}\left(s_{0}, s_{1}\right)(\sigma, \tau)=\mathbf{r}_{l+1}\left(x_{0}, x_{1}\right)(0, \tau) \text {. }
$$

Then

$$
\begin{aligned}
\mathbf{r}_{k+l+1}\left(s_{0}, s_{1}\right)(\sigma, \tau, 0) & =\mathbf{r}_{k+l}\left(s_{0}, s_{1}\right)(\sigma, \tau) \\
& =\mathbf{r}_{l+1}\left(x_{0}, x_{1}\right)(0, \tau)=\mathbf{r}_{l+2}\left(x_{0}, x_{1}\right)(0, \tau, 0)
\end{aligned}
$$

and

$$
\begin{aligned}
\mathbf{r}_{k+l+1}\left(s_{0}, s_{1}\right)(\sigma, \tau, 1) & =\mathbf{r}_{k+l}\left(s_{0}, s_{1}\right)(\sigma, \tau)+\mathbf{r}_{k+l}\left(s_{0}, s_{1}\right)(1-\sigma, 1-\tau) \\
& =\mathbf{r}_{l+1}\left(x_{0}, x_{1}\right)(0, \tau)+\mathbf{r}_{l+1}\left(x_{1}, x_{0}\right)(0,1-\tau) \\
& =\mathbf{r}_{l+1}\left(x_{0}, x_{1}\right)(0, \tau)+\mathbf{r}_{l+1}\left(x_{0}, x_{1}\right)(1,1-\tau) \\
& =\mathbf{r}_{l+2}\left(x_{0}, x_{1}\right)(0, \tau, 1),
\end{aligned}
$$

using (26).

By Lemma 4.4 and Lemma 4.5

$$
\mathbf{r}_{k}\left(s_{0}, s_{1}\right)\left(\sigma_{1}, \sigma_{2}, \ldots, \sigma_{k}\right)=s_{1} \mathbf{r}_{k}\left(\sigma_{1}, \sigma_{2}, \ldots, \sigma_{k}\right)+s_{0} \mathbf{r}_{k}\left(1-\sigma_{1}, \sigma_{2}, \ldots, \sigma_{k}\right)
$$

with

$$
\mathbf{r}_{k}: \mathbf{G}_{k} \rightarrow \mathbb{N}_{0}, \quad \mathbf{r}_{k}:=\mathbf{r}_{k}(0,1) .
$$

These auxiliary coefficients meet the following relation: 
Lemma 4.7. For $k \in \mathbb{N}$ and $\left(\sigma_{1}, \ldots, \sigma_{k}\right) \in \mathbf{G}_{k}$

$$
\mathbf{r}_{k}\left(\sigma_{1}, \ldots, \sigma_{k}\right)=\mathbf{r}_{k+1}\left(\sigma_{1}, \sigma_{1}, \ldots, \sigma_{k}\right)
$$

and

$$
\mathbf{r}_{k}\left(\sigma_{1}, \ldots, \sigma_{k}\right)=\mathbf{r}_{k}\left(\sigma_{1}, \sigma_{k}+\sigma_{1}, \ldots, \sigma_{2}+\sigma_{1}\right) .
$$

Proof. For $k=1$ we have $\mathbf{r}_{2}(\sigma, \sigma)=\mathbf{r}_{1}(\sigma)=\sigma$. Thus by (26)

$$
\begin{aligned}
\mathbf{r}_{k+1}\left(\sigma_{1}, \sigma_{1}, \ldots, \sigma_{k}\right) & =\mathbf{r}_{k}\left(\mathbf{r}_{2}\left(\sigma_{1}, \sigma_{1}\right), \mathbf{r}_{2}\left(1-\sigma_{1}, 1-\sigma_{1}\right)\right)\left(0, \sigma_{2}, \ldots, \sigma_{k}\right) \\
& =\mathbf{r}_{k}\left(\sigma_{1}, 1-\sigma_{1}\right)\left(0, \sigma_{2}, \ldots, \sigma_{k}\right) \\
& =\mathbf{r}_{k}\left(1-\sigma_{1}, \sigma_{1}\right)\left(1, \sigma_{2}, \ldots, \sigma_{k}\right) \\
& =\mathbf{r}_{k}(0,1)\left(\sigma_{1}, \sigma_{2}, \ldots, \sigma_{k}\right)=\mathbf{r}_{k}\left(\sigma_{1}, \sigma_{2}, \ldots, \sigma_{k}\right),
\end{aligned}
$$

using (26) for the third equation. This proves (28).

Equation (29) is automatically met if $k=1$.

We assume that for all $\left(\sigma_{1}, \ldots, \sigma_{k}\right) \in \mathbf{G}_{k}$

$$
\mathbf{r}_{k}\left(\sigma_{1}, \ldots, \sigma_{k}\right)=\mathbf{r}_{k}\left(\sigma_{1}, \sigma_{k}+\sigma_{1}, \ldots, \sigma_{2}+\sigma_{1}\right) .
$$

Then for $\sigma_{k+1}=0$

$$
\begin{aligned}
\mathbf{r}_{k+1}\left(\sigma_{1}, \ldots, \sigma_{k}, 0\right) & =\mathbf{r}_{k}\left(\sigma_{1}, \ldots, \sigma_{k}\right) \\
& =\mathbf{r}_{k}\left(\sigma_{1}, \sigma_{k}+\sigma_{1}, \ldots, \sigma_{2}+\sigma_{1}\right) \\
& =\mathbf{r}_{k+1}\left(\sigma_{1}, 0+\sigma_{1}, \sigma_{k}+\sigma_{1}, \ldots, \sigma_{2}+\sigma_{1}\right),
\end{aligned}
$$

using (28).

$$
\begin{aligned}
& \text { For } \sigma_{k+1}=1 \\
& \qquad \begin{aligned}
\mathbf{r}_{k+1}\left(\sigma_{1}, \ldots, \sigma_{k}, 1\right)= & \mathbf{r}_{k}\left(\sigma_{1}, \ldots, \sigma_{k}\right)+\mathbf{r}_{k}\left(1-\sigma_{1}, \ldots, 1-\sigma_{k}\right) \\
= & \mathbf{r}_{k}\left(\sigma_{1}, \sigma_{k}+\sigma_{1}, \ldots, \sigma_{2}+\sigma_{1}\right) \\
& +\mathbf{r}_{k}\left(1-\sigma_{1}, \sigma_{k}+\sigma_{1}, \ldots, \sigma_{2}+\sigma_{1}\right) \\
= & \mathbf{r}_{k+1}\left(\sigma_{1}, 1+\sigma_{1}, \sigma_{k}+\sigma_{1}, \ldots, \sigma_{2}+\sigma_{1}\right) \\
= & \mathbf{r}_{k+1}\left(\sigma_{1}, \sigma_{k+1}+\sigma_{1}, \sigma_{k}+\sigma_{1}, \ldots, \sigma_{2}+\sigma_{1}\right) .
\end{aligned}
\end{aligned}
$$

For the third equation we used the relation

$$
\mathbf{r}_{l+1}\left(\sigma_{1}, \tau\right)+\mathbf{r}_{l+1}\left(1-\sigma_{1}, \tau\right)=\mathbf{r}_{l+2}\left(\sigma_{1}, 1-\sigma_{1}, \tau\right),
$$

which is valid for $\sigma_{1} \in \mathbf{G}_{1}$ and $\tau \in \mathbf{G}_{l}$ since by (27), (25) and (26)

$$
\begin{aligned}
\mathbf{r}_{l+2}\left(\sigma_{1}, 1-\sigma_{1}, \tau\right) & =\mathbf{r}_{l+1}\left(\mathbf{r}_{2}\left(\sigma_{1}, 1-\sigma_{1}\right), \mathbf{r}_{2}\left(1-\sigma_{1}, \sigma_{1}\right)\right)(0, \tau) \\
& =\mathbf{r}_{l+1}(1,1)(0, \tau)=\mathbf{r}_{l+1}(0,1)(0, \tau)+\mathbf{r}_{l+1}(1,0)(0, \tau) \\
& =\mathbf{r}_{l+1}(0,1)(0, \tau)+\mathbf{r}_{l+1}(0,1)(1, \tau) \\
& =\mathbf{r}_{l+1}(0, \tau)+\mathbf{r}_{l+1}(1, \tau)
\end{aligned}
$$

This proves (29).

Equation (29) implies the following symmetry of the grand canonical energy function: 
Lemma 4.8. For $\left(\sigma_{1}, \ldots, \sigma_{k}\right) \in \mathbf{G}_{k}$

$$
\mathbf{H}_{k}^{G}\left(\sigma_{k}, \ldots, \sigma_{1}\right)=\mathbf{H}_{k}^{G}\left(\sigma_{1}, \ldots, \sigma_{k}\right)
$$

Proof. By definition,

$$
\mathbf{H}_{k}^{G}\left(\sigma_{1}, \ldots, \sigma_{k}\right)=\mathbf{H}_{k+1}^{C}\left(\sigma_{1}, \ldots, \sigma_{k}, 1\right)=\ln \left(\mathbf{h}_{k+1}^{C}\left(\sigma_{1}, \ldots, \sigma_{k}, 1\right)\right) .
$$

So we must show that

$$
\mathbf{h}_{k+1}^{C}\left(\sigma_{1}, \ldots, \sigma_{k}, 1\right)=\mathbf{h}_{k+1}^{C}\left(\sigma_{k}, \ldots, \sigma_{1}, 1\right) .
$$

The functions $\mathbf{r}_{k}: \mathbf{G}_{k} \rightarrow \mathbb{N}_{0}$ are related to the functions $\mathbf{h}_{k}^{C}$ by

$$
\mathbf{h}_{k}^{C}\left(\sigma_{1}, \ldots, \sigma_{k}\right)=\mathbf{r}_{k+2}\left(\sigma, 1-\sigma, \sigma_{1}, \ldots, \sigma_{k}\right)
$$

for $\sigma=0$ or 1 , since

$$
\mathbf{h}_{0}^{c}=1=\mathbf{r}_{2}(0,1)=\mathbf{r}_{2}(1,0)
$$

and by induction in $k$.

(29) implies (31), since by (32)

$$
\begin{aligned}
\mathbf{h}_{k+1}^{C}\left(\sigma_{1}, \ldots, \sigma_{k}, 1\right) & =\mathbf{r}_{k+3}\left(0,1, \sigma_{1}, \ldots, \sigma_{k}, 1\right)=\mathbf{r}_{k+3}\left(0,1, \sigma_{k}, \ldots, \sigma_{1}, 1\right) \\
& =\mathbf{h}_{k+1}^{C}\left(\sigma_{k}, \ldots, \sigma_{1}, 1\right) . \square
\end{aligned}
$$

Lemma (4.8) implies the following mirror symmetry of the grand canonical interaction coefficients:

Proposition 4.9. Let $\left(t_{1}, \ldots, t_{k}\right) \in \mathbf{G}_{k}^{*}$. Then

$$
j_{k}^{G}\left(t_{1}, \ldots, t_{k}\right)=j_{k}^{G}\left(t_{k}, \ldots, t_{1}\right) .
$$

Proof. This follows immediately from (30), since

$$
\begin{aligned}
j_{k}^{G}\left(t_{1}, \ldots, t_{k}\right) & =-2^{-k} \sum_{\left(\sigma_{1}, \ldots, \sigma_{k}\right) \in \mathbf{G}_{k}} \mathbf{H}_{k}^{G}\left(\sigma_{1}, \ldots, \sigma_{k}\right) \chi_{t}\left(\sigma_{1}, \ldots, \sigma_{k}\right) \\
& =-2^{-k} \sum_{\left(\sigma_{1}, \ldots, \sigma_{k}\right) \in \mathbf{G}_{k}} \mathbf{H}_{k}^{G}\left(\sigma_{k}, \ldots, \sigma_{1}\right) \chi_{\left(t_{1}, \ldots, t_{k}\right)}\left(\sigma_{1}, \ldots, \sigma_{k}\right) \\
& =-2^{-k} \sum_{\left(\sigma_{1}, \ldots, \sigma_{k}\right) \in \mathbf{G}_{k}} \mathbf{H}_{k}^{G}\left(\sigma_{k}, \ldots, \sigma_{1}\right) \chi_{\left(t_{k}, \ldots, t_{1}\right)}\left(\sigma_{k}, \ldots, \sigma_{1}\right) \\
& =j_{k}^{G}\left(t_{k}, \ldots, t_{1}\right) .
\end{aligned}
$$

\section{Ferromagnetism}

In this section we will show that the canonical and grand canonical interaction is ferromagnetic in the weak sense that for all $k \in \mathbb{N}$

$$
j_{k}^{C}(t) \geqq 0 \quad \text { and } \quad j_{k}^{G}(t) \geqq 0 \quad \text { for } t \in \mathbf{G}_{k} \backslash\{0\} .
$$

That property will be a consequence of inequalities between the interaction coefficients for spin chains of length $k$ and length $k+1$. These inequalities are derived by interpolation techniques. 
We begin with the canonical ensemble. For $k \in \mathbb{N}, \sigma=\left(\sigma_{1}, \ldots, \sigma_{k}\right) \in \mathbf{G}_{k}$ and $0 \leqq u \leqq 1$ let

$$
\mathbf{h}_{k}^{C}(u ; \sigma):=u \mathbf{h}_{k}^{C}(\sigma)+(1-u) \mathbf{h}_{k-1}^{C}\left(\sigma_{2} \ldots, \sigma_{k}\right)
$$

and $\mathbf{H}_{k}^{C}(u ; \sigma):=\ln \left(\mathbf{h}_{k}^{C}(u ; \sigma)\right)$.

For the interpolating canonical interaction coefficients

$$
j_{k}^{C}(u ; t):=-\mathscr{F}_{k}\left(\mathbf{H}_{k}^{C}(u)\right)(t), \quad t=\left(t_{1}, \ldots, t_{k}\right) \in \mathbf{G}_{k}^{*},
$$

we have

$$
j_{k}^{C}(0 ; t)= \begin{cases}0, & t_{1}=1 \\ j_{k-1}^{C}\left(t_{2}, \ldots, t_{k}\right), & t_{1}=0\end{cases}
$$

and

$$
j_{k}^{C}(1 ; t)=j_{k}^{C}(t)
$$

We will show that $\frac{d}{d u} j_{k}^{c}(u ;(s, 1)) \geqq 0$ for $s \in \mathbf{G}_{k-1}^{*}$ and $0 \leqq u \leqq 1$. The canonical interaction coefficients $j_{k}^{C}(t)$ for $t \in \mathbf{G}_{k}^{*}$ of the form $t=(s, 0)$ will be discussed later.

For $k=1$ we have

$$
\frac{d}{d u} j_{1}^{c}(u ; 1)=\frac{1}{2} \frac{d}{d u} \ln (2 u+1-u)=\frac{1}{2}(1+u)^{-1}>0 .
$$

We show

$$
\frac{d}{d u} j_{k+1}^{c}(u ;(s, 1)) \geqq 0, \quad u \in[0,1]
$$

for $s \in \mathbf{G}_{k}^{*}$, assuming that $\frac{d}{d u} j_{k}^{c}\left(u ;\left(s^{\prime}, 1\right)\right) \geqq 0$ for all $s^{\prime} \in \mathbf{G}_{k-1}^{*}$.

For $\#_{k}(s)$ odd we have

$$
\begin{aligned}
j_{k+1}^{C}(u ;(s, 1)) & =-2^{-(k+1)} \sum_{\sigma \in \mathbf{G}_{k}}\left(\mathbf{H}_{k+1}^{C}(u ;(\sigma, 0))-\mathbf{H}_{k+1}^{C}(u ;(\sigma, 1))\right) \chi_{s}(\sigma) \\
& =-2^{-(k+1)} \sum_{\sigma \in \mathbf{G}_{k}} \mathbf{H}_{k+1}^{C}(u ;(\sigma, 0)) \chi_{s}(\sigma) \\
& \left.=-2^{-(k+1)} \sum_{\sigma \in \mathbf{G}_{k}} \mathbf{H}_{k}^{C}(u ; \sigma)\right) \chi_{s}(\sigma)=\frac{1}{2} j_{k}^{C}(u ; s),
\end{aligned}
$$

since $\mathbf{H}_{k+1}^{C}(u ; 1-\sigma, 1)=\mathbf{H}_{k+1}^{C}(u ; \sigma, 1)$ and $\chi_{s}(1-\sigma)=-\chi_{s}(\sigma)$, showing (35) for $\#_{k}(s)$ odd.

Therefore we assume $\#_{k}(s)$ to be even.

We write

$$
j_{k+1}^{C}(u ;(s, 1))=-2^{-(k+1)} \sum_{\sigma \in \mathbf{G}_{k}} \ln \left(\mathbf{v}_{k}(u ; \sigma)\right) \chi_{s}(\sigma)
$$

with $\mathbf{v}_{k}(u ; \sigma)$ defined for $k \in \mathbb{N}_{0}, u \in[0,1]$ and $\sigma \in \mathbf{G}_{k}$ by

$$
\mathbf{v}_{k}(u ; \sigma):=\frac{\mathbf{h}_{k+1}^{C}(u ;(\sigma, 0))}{\mathbf{h}_{k+1}^{C}(u ;(\sigma, 1))} .
$$


We have

$$
\mathbf{v}_{k}(u ; 1-\sigma)=1-\mathbf{v}_{k}(u ; \sigma),
$$

since $\mathbf{h}_{k+1}^{C}(u ;(\sigma, 1))=\mathbf{h}_{k}^{C}(u ; \sigma)+\mathbf{h}_{k}^{C}(u ; 1-\sigma)$.

Moreover, $\mathbf{v}_{k}(u)$ meets the recursion relations

$$
\mathbf{v}_{k+1}(u ;(\sigma, 0))=\frac{\mathbf{v}_{k}(u ; \sigma)}{1+\mathbf{v}_{k}(u ; \sigma)} \quad \text { and } \quad \mathbf{v}_{k+1}(u ;(\sigma, 1))=\frac{1}{1+\mathbf{v}_{k}(u ; 1-\sigma)},
$$

since

$$
\begin{aligned}
\mathbf{v}_{k+1}(u ;(\sigma, 0)) & =\frac{\mathbf{h}_{k+1}^{C}(u ;(\sigma, 0))}{\mathbf{h}_{k+1}^{C}(u ;(\sigma, 0))+\mathbf{h}_{k+1}^{C}(u ;(1-\sigma, 1))} \\
& =\frac{\mathbf{h}_{k+1}^{C}(u ;(\sigma, 0))}{\mathbf{h}_{k+1}^{C}(u ;(\sigma, 0))+\mathbf{h}_{k+1}^{C}(u ;(\sigma, 1))},
\end{aligned}
$$

and by (38). We derive (36) and get with $s^{\prime}:=\left(s_{1}, \ldots, s_{k-1}\right)$

$$
\begin{aligned}
& \frac{d}{d u} j_{k+1}^{C}(u ;(s, 1)) \\
& \quad=-2^{-(k+1)} \sum_{\sigma \in \mathbf{G}_{k-1}} \frac{d}{d u}\left[\ln \left(\mathbf{v}_{k}(u ;(\sigma, 0))\right)+(-1)^{s_{k}} \ln \left(\mathbf{v}_{k}(u ;(\sigma, 1))\right)\right] \chi_{s^{\prime}}(\sigma) \\
& =-2^{-(k+1)} \sum_{\sigma \in \mathbf{G}_{k^{-1}}} \frac{d}{d u}\left[\ln \left(\mathbf{v}_{k}(u ;(\sigma, 0))\right)+\ln \left(\mathbf{v}_{k}(u ;(1-\sigma, 1))\right)\right] \chi_{s^{\prime}}(\sigma) \\
& =-2^{-(k+1)} \sum_{\sigma \in \mathbf{G}_{k-1}} \frac{d}{d u} \ln \left[\frac{\mathbf{v}_{k-1}(u ; \sigma)}{\left(1+\mathbf{v}_{k-1}(u ; \sigma)\right)^{2}}\right] \chi_{s^{\prime}}(\sigma) \\
& \quad=-2^{-(k+1)} \sum_{\sigma \in \mathbf{G}_{k-1}} \frac{1-\mathbf{v}_{k-1}(u ; \sigma)}{1+\mathbf{v}_{k-1}(u ; \sigma)} \cdot \frac{d}{d u} \ln \left(\mathbf{v}_{k-1}(u ; \sigma)\right) \chi_{s^{\prime}}(\sigma) .
\end{aligned}
$$

The second equation follows from the relation

$$
\chi_{\left(s_{1}, \ldots, s_{k-1}\right)}(1-\sigma)=(-1)^{s_{k}} \chi_{\left(s_{1}, \ldots, s_{k-1}\right)}(\sigma)
$$

which is valid since $\#_{k}(s)$ is even.

Therefore

$$
\begin{aligned}
& \frac{d}{d u} j_{k+1}^{C}(u ;(s, 1)) \\
& =\frac{1}{2} \mathscr{F}_{k-1}\left[\frac{1-\mathbf{v}_{k-1}(u ; \cdot)}{1+\mathbf{v}_{k-1}(u ; \cdot)} \cdot\left(-\frac{d}{d u} \ln \left(\mathbf{v}_{k-1}(u ; \cdot)\right)\right)\right]\left(s_{1}, \ldots, s_{k-1}\right),
\end{aligned}
$$

that is, the left hand side is half the Fourier transform of the product of two functions in $\mathscr{A}_{k-1}$.

By our assumption $\frac{d}{d u} j_{k}^{c}\left(u ;\left(s^{\prime}, 1\right)\right) \geqq 0$ the second function is strictly ferromagnetic. So in order to prove (35) for $\#_{k}(s)$ even, we may prove that the first function is strictly ferromagnetic, too. 
Lemma 5.1. For $k \in \mathbb{N}_{0}$ and $u \in[0,1]$

$$
\frac{1-\mathbf{v}_{k}(u ; \cdot)}{1+\mathbf{v}_{k}(u ; \cdot)} \in \mathscr{C}_{k} .
$$

Proof.

$$
\begin{aligned}
\frac{1-}{1+} & \mathbf{v}_{k}(u ; \sigma) \\
& =\frac{\mathbf{h}_{k+1}^{C}(u ; \sigma)}{\mathbf{h}_{k+1}^{C}(u ;(\sigma, 1))+\mathbf{h}_{k+1}^{C}(u ;(\sigma, 0))} \\
& =\frac{\mathbf{h}_{k}^{C}(u ; \sigma)+\mathbf{h}_{k}^{C}(u ; 1-\sigma)-\mathbf{h}_{k}^{C}(u ; \sigma)}{\mathbf{h}_{k+1}^{C}(u ;(1-\sigma, 1))+\mathbf{h}_{k+1}^{C}(u ;(\sigma, 0))}=\frac{\mathbf{h}_{k}^{C}(u ; 1-\sigma)}{\mathbf{h}_{k+2}^{C}(u ;(\sigma, 0,1))} \\
& =\frac{u \mathbf{h}_{k}^{C}(1-\sigma)+(1-u) \mathbf{h}_{k-1}^{C}\left(1-\sigma_{2}, \ldots, 1-\sigma_{k}\right)}{u \mathbf{h}_{k+2}^{C}(\sigma, 0,1)+(1-u) \mathbf{h}_{k+1}^{C}\left(\sigma_{2}, \ldots, \sigma_{k}, 0,1\right)} \\
= & \frac{u \mathbf{r}_{k+2}(0,1,1-\sigma)+(1-u) \mathbf{r}_{k+1}\left(0,1,1-\sigma_{2}, \ldots, 1-\sigma_{k}\right)}{u \mathbf{r}_{k+4}(0,1, \sigma, 0,1)+(1-u) \mathbf{r}_{k+3}\left(0,1, \sigma_{2}, \ldots, \sigma_{k}, 0,1\right)} \\
= & \frac{u \mathbf{r}_{k+2}\left(0,1-\sigma_{k}, \ldots, 1-\sigma_{1}, 1\right)+(1-u) \mathbf{r}_{k+1}\left(0,1-\sigma_{k}, \ldots, 1-\sigma_{2}, 1\right)}{u \mathbf{r}_{k+4}\left(0,1,0, \sigma_{k}, \ldots, \sigma_{1}, 1\right)+(1-u) \mathbf{r}_{k+3}\left(0,1,0, \sigma_{k}, \ldots, \sigma_{2}, 1\right)} \\
= & \frac{u \mathbf{r}_{k+2}\left(1, \sigma_{k}, \ldots, \sigma_{1}, 1\right)+(1-u) \mathbf{r}_{k+1}\left(1, \sigma_{k}, \ldots, \sigma_{2}, 1\right)}{u \mathbf{r}_{k+4}\left(0,1,0, \sigma_{k}, \ldots, \sigma_{1}, 1\right)+(1-u) \mathbf{r}_{k+3}\left(0,1,0, \sigma_{k}, \ldots, \sigma_{2}, 1\right)} \\
= & \frac{\mathbf{W}_{k}\left(u ; \mathbf{r}_{1}(1), \mathbf{r}_{1}(0)\right)(\sigma)}{\mathbf{W}_{k}\left(u ; \mathbf{r}_{3}(0,1,0), \mathbf{r}_{3}(1,0,1)\right)(\sigma)}=\frac{\mathbf{W}_{k}(u ; 1,0)(\sigma)}{\mathbf{W}_{k}(u ; 1,2)(\sigma)}
\end{aligned}
$$

with

$$
\begin{aligned}
\mathbf{W}_{k}\left(u ; s_{0}, s_{1}\right)(\sigma):= & u \mathbf{r}_{k+2}\left(s_{0}, s_{1}\right)\left(0, \sigma_{k}, \ldots, \sigma_{1}, 1\right) \\
& +(1-u) \mathbf{r}_{k+1}\left(s_{0}, s_{1}\right)\left(0, \sigma_{k}, \ldots, \sigma_{2}, 1\right)
\end{aligned}
$$

for $u \in[0,1], s_{0}, s_{1} \in \mathbb{R}$ and $\sigma=\left(\sigma_{1}, \ldots, \sigma_{k}\right) \in \mathbf{G}_{k}$.

To derive the first equation in this long train of derivations, we used the definition (37) of $\mathbf{v}_{k}(u)$.

Equation 5 follows from the relations (32) between $\mathbf{h}_{k}^{C}$ and the auxiliary coefficients $\mathbf{r}_{k}$.

For Eqs. 6 and 7 we used Lemma 4.7 and the relation

$$
\mathbf{r}_{k+1}(\tau, 1)=\mathbf{r}_{k+1}(1-\tau, 1)
$$

valid for all $\tau \in \mathbf{G}_{k}$.

The second-to-last equality is obtained using Lemma 4.6.

Now we will consider general expressions of the form

$$
\frac{\mathbf{W}_{l}\left(u ; n_{0}, n_{1}\right)(\sigma)}{\mathbf{W}_{l}\left(u ; d_{0}, d_{1}\right)(\sigma)}
$$


For fixed arguments, numerator and denominator of (40) may be considered to be ordinate and abscissa of a vector in $\mathbb{R}^{2}$. Then (40) is the tangent of its angle with the abscissa.

By applying linear transformations in $\mathbb{R}^{2}$ and using Proposition 3.2, we show now that for $k \in \mathbb{N}, n_{0}, d_{0}, d_{1} \in \mathbb{R}^{+}$and $u \in[0,1]$

$$
\frac{\mathbf{W}_{k}\left(u ; n_{0}, 0\right)}{\mathbf{W}_{k}\left(u ; d_{0}, d_{1}\right)} \in \mathscr{C}_{k} \quad \text { if } d_{0}<d_{1},
$$

that is, the function is strictly ferromagnetic.

$$
\frac{\mathbf{W}_{k}\left(u ; n_{0}, 0\right)}{\mathbf{W}_{k}\left(u ; s_{0}, s_{1}\right)}=\lambda \frac{\mathbf{W}_{k}\left(u ; \tilde{n}_{0}, 0\right)}{\mathbf{W}_{k}\left(u ; s_{0}, s_{1}\right)}
$$

with $\tilde{n}_{0}:=\sqrt{d_{1}^{2}-d_{0}^{2}}$ and $\lambda:=n_{0} / \tilde{n}_{0}>0$, so that the first quotient is strictly ferromagnetic if the second is.

With $n^{\prime}:=\sqrt{d_{1}\left(d_{1}-d_{0}\right) / 2}$ and $d^{\prime}:=\sqrt{d_{1}\left(d_{1}+d_{0}\right) / 2}$

$$
\left(\begin{array}{c}
\mathbf{W}_{k}\left(u ; \tilde{n}_{0}, 0\right) \\
\mathbf{W}_{k}\left(u ; d_{0}, d_{1}\right)
\end{array}\right)=\left(\begin{array}{cc}
\sqrt{\frac{d_{1}+d_{0}}{2 d_{1}}} & \sqrt{\frac{d_{1}-d_{0}}{2 d_{1}}} \\
-\sqrt{\frac{d_{1}-d_{0}}{2 d_{1}}} & \sqrt{\frac{d_{1}+d_{0}}{2 d_{1}}}
\end{array}\right)\left(\begin{array}{c}
\mathbf{W}_{k}\left(u ; n^{\prime},-n^{\prime}\right) \\
\mathbf{W}_{k}\left(u ; d^{\prime}, d^{\prime}\right)
\end{array}\right),
$$

since

$$
\begin{aligned}
& \sqrt{\frac{d_{1}+d_{0}}{2 d_{1}}} \mathbf{W}_{k}\left(u ; n^{\prime},-n^{\prime}\right)+\sqrt{\frac{d_{1}-d_{0}}{2 d_{1}}} \mathbf{W}_{k}\left(u ; d^{\prime}, d^{\prime}\right) \\
& =n^{\prime} \sqrt{\frac{d_{1}+d_{0}}{2 d_{1}}}\left(\mathbf{W}_{k}(u ; 1,0)-\mathbf{W}_{k}(u ; 0,1)\right) \\
& \quad+d^{\prime} \sqrt{\frac{d_{1}-d_{0}}{2 d_{1}}}\left(\mathbf{W}_{k}(u ; 1,0)+\mathbf{W}_{k}(u ; 0,1)\right) \\
& =\frac{\sqrt{d_{1}^{2}-d_{0}^{2}}}{2} \cdot 2 \mathbf{W}_{k}(u ; 1,0)=\mathbf{W}_{k}\left(u ; \sqrt{d_{1}^{2}-d_{0}^{2}}, 0\right)
\end{aligned}
$$

and

$$
\begin{gathered}
-\sqrt{\frac{d_{1}-d_{0}}{2 d_{1}}} \mathbf{W}_{k}\left(u ; n^{\prime},-n^{\prime}\right)+\sqrt{\frac{d_{1}+d_{0}}{2 d_{1}}} \mathbf{W}_{k}\left(u ; d^{\prime}, d^{\prime}\right) \\
=-\frac{d_{1}-d_{0}}{2}\left(\mathbf{W}_{k}(u ; 1,0)-\mathbf{W}_{k}(u ; 0,1)\right) \\
\quad+\frac{d_{1}+d_{0}}{2}\left(\mathbf{W}_{k}(u ; 1,0)+\mathbf{W}_{k}(u ; 0,1)\right) \\
=d_{0} \mathbf{W}_{k}(u ; 1,0)+d_{1} \mathbf{W}_{k}(u ; 0,1)=\mathbf{W}_{k}\left(u ; d_{0}, d_{1}\right) .
\end{gathered}
$$


Furthermore,

$$
\begin{aligned}
\frac{\mathbf{W}_{k}\left(u ; \tilde{n}_{0}, 0\right)}{\mathbf{W}_{k}\left(u ; d_{0}, d_{1}\right)} & =\frac{\sqrt{d_{1}+d_{0}} \mathbf{W}_{k}\left(u ; n^{\prime},-n^{\prime}\right)+\sqrt{d_{1}-d_{0}} \mathbf{W}_{k}\left(u ; d^{\prime}, d^{\prime}\right)}{-\sqrt{d_{1}-d_{0}} \mathbf{W}_{k}\left(u ; n^{\prime},-n^{\prime}\right)+\sqrt{d_{1}+d_{0}} \mathbf{W}_{k}\left(u ; d^{\prime}, d^{\prime}\right)} \\
& =\mathrm{g}_{y}\left(\frac{\mathbf{W}_{k}\left(u ; n^{\prime},-n^{\prime}\right)}{\mathbf{W}_{k}\left(u ; d^{\prime}, d^{\prime}\right)}\right),
\end{aligned}
$$

with $\mathrm{g}_{z}:(-1 / z, 1 / z) \rightarrow \mathbb{R}$ defined for $z \in \mathbb{R}^{+}$by

$$
\mathrm{g}_{z}(x):=\frac{x+z}{1-z x}
$$

for $y:=\sqrt{d_{1}^{2}-d_{0}^{2}} /\left(d_{1}+d_{0}\right)=\tilde{n}_{0} /\left(d_{1}+d_{0}\right)<1$. Now the $n$-th derivatives of $\mathrm{g}_{z}$, are positive at zero,

$$
\left.\frac{d^{n}}{d x^{n}} \mathbf{g}_{z}(x)\right|_{x=0} \geqq 0, \quad n \in \mathbb{N}_{0},
$$

since both numerator and the inverse denominator of $\mathrm{g}_{z}$ have positive $x$-derivatives. Moreover, $g_{z}$ is real-analytic for arguments of norm $|x|<1 / z$, so that we can apply Proposition 3.2. Thus the 1.h.s. of (42) is a strictly ferromagnetic observable in $\mathscr{A}_{k}$ if the argument of $\mathrm{g}_{z}$ has the same property.

But for $c \in \mathbb{R}$

$$
\mathbf{W}_{k}(u ; c, \pm c)(1-\sigma)= \pm \mathbf{W}_{k}(u ; c, \pm c)(\sigma)
$$

since

$$
\begin{aligned}
& \mathbf{W}_{k}(u ; c, \pm c)(1-\sigma)=c\left(\mathbf{W}_{k}(u ; 1,0)(1-\sigma) \pm \mathbf{W}_{k}(u ; 0,1)(1-\sigma)\right) \\
& =c\left[u \left(\mathbf{r}_{k+2}(0,1)\left(1,1-\sigma_{k}, \ldots, 1-\sigma_{1}, 1\right)\right.\right. \\
& \left.\quad \pm \mathbf{r}_{k+2}(0,1)\left(0,1-\sigma_{k}, \ldots, 1-\sigma_{1}, 1\right)\right) \\
& +(1-u)\left(\mathbf{r}_{k+1}(0,1)\left(1,1-\sigma_{k}, \ldots, 1-\sigma_{2}, 1\right)\right. \\
& \left.\left.\quad \pm \mathbf{r}_{k+1}(0,1)\left(0,1-\sigma_{k}, \ldots, 1-\sigma_{2}, 1\right)\right)\right] \\
& =c\left[u\left(\mathbf{r}_{k+2}(0,1)\left(0, \sigma_{k}, \ldots, \sigma_{1}, 1\right) \pm \mathbf{r}_{k+2}(0,1)\left(1, \sigma_{k}, \ldots, \sigma_{1}, 1\right)\right)\right. \\
& \quad \quad+(1-u)\left(\mathbf{r}_{k+1}(0,1)\left(0, \sigma_{k}, \ldots, \sigma_{2}, 1\right)\right. \\
& \left.\left.\quad \quad \pm \mathbf{r}_{k+1}(0,1)\left(1, \sigma_{k}, \ldots, \sigma_{2}, 1\right)\right)\right] \\
& = \pm \mathbf{W}_{k}(u ; c, \pm c)(\sigma) .
\end{aligned}
$$

Thus for $\#_{k}(t)$ even

$$
\mathscr{F}_{k}\left(\frac{\mathbf{W}_{k}\left(u ; n^{\prime},-n^{\prime}\right)}{\mathbf{W}_{k}\left(u ; d^{\prime}, d^{\prime}\right)}\right)(t)=0,
$$

since the quotient is odd w.r.t. inversion of all spins.

Therefore we consider the case of an odd $\#_{k}(t)$. If $k=1$, then

$$
\mathscr{F}_{1}\left(\frac{\mathbf{W}_{1}\left(u ; n^{\prime},-n^{\prime}\right)}{\mathbf{W}_{1}\left(u ; d^{\prime}, d^{\prime}\right)}\right)(1)=\frac{u}{2+u} \frac{n^{\prime}}{d^{\prime}} \geqq 0 \quad \text { for } u \in[0,1] .
$$


If $k>1$, then for $\#_{k}(t)$ odd

$$
\begin{aligned}
\mathscr{F}_{k}\left(\frac{\mathbf{W}_{k}\left(u ; n^{\prime},-n^{\prime}\right)}{\mathbf{W}_{k}\left(u ; d^{\prime}, d^{\prime}\right)}\right)(t) & =2^{-k} \sum_{\sigma \in \mathbf{G}_{k-1}} 2 \cdot \frac{\mathbf{W}_{k}\left(u ; n^{\prime},-n^{\prime}\right)(\sigma, 0)}{\mathbf{W}_{k}\left(u ; d^{\prime}, d^{\prime}\right)(\sigma, 0)} \chi_{\left(t_{1}, \ldots, t_{k-1}\right)}(\sigma) \\
& =\mathscr{F}_{k-1}\left(\frac{\mathbf{W}_{k-1}\left(u ; n^{\prime}, 0\right)}{\mathbf{W}_{k-1}\left(u ; d^{\prime}, 2 d^{\prime}\right)}\right)\left(t_{1}, \ldots, t_{k-1}\right) .
\end{aligned}
$$

The first equation of (44) is a consequence of (43). The second equation is derived as follows.

$$
\begin{aligned}
& \quad \mathbf{W}_{k}\left(u ; n^{\prime},-n^{\prime}\right)(\sigma, 0)=u \mathbf{r}_{k+2}\left(n^{\prime},-n^{\prime}\right)\left(0,0, \sigma_{k-1}, \ldots, \sigma_{1}, 1\right) \\
& +(1-u) \mathbf{r}_{k+1}\left(n^{\prime},-n^{\prime}\right)\left(0,0, \sigma_{k-1}, \ldots, \sigma_{2}, 1\right) \\
& =u \mathbf{r}_{k+1}\left(\mathbf{r}_{2}\left(n^{\prime},-n^{\prime}\right)(0,0), \mathbf{r}_{2}\left(n^{\prime},-n^{\prime}\right)(1,1)\right)\left(0, \sigma_{k-1}, \ldots, \sigma_{1}, 1\right) \\
& +(1-u) \mathbf{r}_{k}\left(\mathbf{r}_{2}\left(n^{\prime},-n^{\prime}\right)(0,0), \mathbf{r}_{2}\left(n^{\prime},-n^{\prime}\right)(1,1)\right)\left(0, \sigma_{k-1}, \ldots, \sigma_{2}, 1\right) \\
& =u \mathbf{r}_{k+1}\left(n^{\prime}, 0\right)\left(0, \sigma_{k-1}, \ldots, \sigma_{1}, 1\right)+(1-u) \mathbf{r}_{k}\left(n^{\prime}, 0\right)\left(0, \sigma_{k-1}, \ldots, \sigma_{2}, 1\right) \\
& =\mathbf{W}_{k-1}\left(u ; n^{\prime}, 0\right)(\sigma),
\end{aligned}
$$

using (27).

Similarly,

$$
\begin{aligned}
\mathbf{W}_{k}\left(u ; d^{\prime}, d^{\prime}\right)(\sigma, 0)= & u \mathbf{r}_{k+1}\left(\mathbf{r}_{2}\left(d^{\prime}, d^{\prime}\right)(0,0), \mathbf{r}_{2}\left(d^{\prime}, d^{\prime}\right)(1,1)\right)\left(0, \sigma_{k-1}, \ldots, \sigma_{1}, 1\right) \\
& +(1-u) \mathbf{r}_{k}\left(\mathbf{r}_{2}\left(d^{\prime}, d^{\prime}\right)(0,0), \mathbf{r}_{2}\left(d^{\prime}, d^{\prime}\right)(1,1)\right)\left(0, \sigma_{k-1}, \ldots, \sigma_{2}, 1\right) \\
= & u \mathbf{r}_{k+1}\left(d^{\prime}, 2 d^{\prime}\right)\left(0, \sigma_{k-1}, \ldots, \sigma_{1}, 1\right) \\
& +(1-u) \mathbf{r}_{k}\left(d^{\prime}, 2 d^{\prime}\right)\left(0, \sigma_{k-1}, \ldots, \sigma_{2}, 1\right) \\
= & \mathbf{W}_{k-1}\left(u ; d^{\prime}, 2 d^{\prime}\right)(\sigma) .
\end{aligned}
$$

The argument of the Fourier transform in (44) is of the form of (40), with $l:=k-1$, $n_{0}:=n^{\prime}, n_{1}:=0, d_{0}:=d^{\prime}$ and $d_{1}:=2 d^{\prime}$. In particular $n_{0}, d_{0}, d_{1} \in \mathbb{R}^{+}$and $d_{0}<d_{1}$ so that we have proven the recursive step $k-1 \rightarrow k$. Thus Eq. (41) holds true.

Now we know that for $k \in \mathbb{N}$ and $s \in \mathbf{G}_{k-1}^{*}$

$$
\frac{d}{d u} j_{k}^{C}(u ;(s, 1)) \geqq 0, \quad u \in[0,1] .
$$

Together with the formulae (33) for $j_{k}^{c}(0 ; t)$ and (34) for $j_{k}^{c}(1 ; t)$ this implies

Corollary 5.2. For $k \in \mathbb{N}$ and $t=\left(t_{1}, \ldots, t_{k}\right) \in \mathbf{G}_{k}^{*}$

$$
j_{k+1}^{C}(t, 1) \geqq \begin{cases}0, & t_{1}=1 \\ j_{k}^{C}\left(t_{2}, \ldots, t_{k}, 1\right), & t_{1}=0 .\end{cases}
$$

We know from Lemma 4.2 that

$$
j_{k+1}^{C}(t, 0)=j_{k+1}^{C}(t, 1)+j_{k}^{G}(t) .
$$

Thus the canonical ensemble is weakly ferromagnetic if the grand canonical ensemble is weakly ferromagnetic, a property which we will show now. 
For $k \in \mathbb{N}_{0}, \sigma \in \mathbf{G}_{k}$ and $u \in[0,1]$ we set

$$
\mathbf{h}_{k}^{G}(u ; \sigma):=u \mathbf{h}_{k+1}^{G}(0, \sigma)+(1-u) \mathbf{h}_{k}^{G}(\sigma)
$$

and

$$
\mathbf{H}_{k}^{G}(u ; \sigma):=\ln \left(\mathbf{h}_{k}^{G}(u ; \sigma)\right) .
$$

For the interpolating grand canonical coefficients

$$
j_{k}^{G}(u ; t):=-\mathscr{F}_{k}\left(\mathbf{H}_{k}^{G}(u)\right)(t), \quad t \in \mathbf{G}_{k}^{*},
$$

we have for $t=\left(t_{1}, \ldots, t_{k}\right)$

$$
j_{k}^{G}(t)=j_{k}^{G}(0 ; t)
$$

and

$$
j_{k+1}^{G}\left(t_{0}, t\right)=j_{k}^{G}(1 ; t), \quad t_{0}:=\#_{k}(t)(\bmod 2),
$$

since

$$
\begin{aligned}
j_{k+1}^{G}\left(t_{0}, t\right) & =-2^{-(k+1)} \sum_{\sigma \in \mathbf{G}_{k+1}} \mathbf{H}_{k+1}^{G}(\sigma) \boldsymbol{\chi}_{\left(t_{0}, t\right)}(\sigma) \\
& =-2^{-(k+1)} \sum_{\tau \in \mathbf{G}_{k}}\left(\mathbf{H}_{k+1}^{G}(0, \tau)+\mathbf{H}_{k+1}^{G}(1,1-\tau)\right) \boldsymbol{\chi}_{t}(\tau) \\
& =-2^{-k} \sum_{\tau \in \mathbf{G}_{k}} \mathbf{H}_{k+1}^{G}(0, \tau) \chi_{t}(\tau)=j_{k}^{G}(1 ; \tau)
\end{aligned}
$$

using $\mathbf{H}_{l}^{G}(1-\sigma)=\mathbf{H}_{l}^{G}(\sigma)$.

One notices that $\#_{k+1}\left(t_{0}, t\right)$ is always even, so that we can obtain information on the complement of those grand canonical coefficients which vanish by Proposition 4.3 .

Lemma 5.3. For $k \in \mathbb{N}_{0}$ and $\sigma=\left(\sigma_{1}, \ldots, \sigma_{k}\right) \in \mathbf{G}_{k}$

$$
\frac{d}{d u} \mathbf{H}_{k}^{G}(u ; \sigma)=\frac{\mathbf{v}_{k}(1 ; \tau)}{1+u \cdot \mathbf{v}_{k}(1 ; \tau)}, \quad u \in[0,1]
$$

with $\tau:=\left(\sigma_{k}, \ldots, \sigma_{1}\right)$.

Proof. By definition (47) of $\mathbf{H}_{k}^{G}(u ; \sigma),(46)$ of $\mathbf{h}_{k}^{G}(u ; \sigma)$ and (24) of $\mathbf{h}_{k}^{G}(\sigma)$ we have

$$
\begin{aligned}
\frac{d}{d u} \mathbf{H}_{k}^{G}(u ; \sigma) & =\frac{d}{d u} \ln \left(\mathbf{h}_{k}^{G}(u ; \sigma)\right)=\frac{\mathbf{h}_{k+1}^{G}(0, \sigma)-\mathbf{h}_{k}^{G}(\sigma)}{\mathbf{h}_{k}^{G}(\sigma)+u\left(\mathbf{h}_{k+1}^{G}(0, \sigma)-\mathbf{h}_{k}^{G}(\sigma)\right)} \\
& =\frac{\mathbf{h}_{k+1}^{G}(\tau, 0)-\mathbf{h}_{k}^{G}(\tau)}{\mathbf{h}_{k}^{G}(\tau)+u\left(\mathbf{h}_{k+1}^{G}(\tau, 0)-\mathbf{h}_{k}^{G}(\tau)\right)} \\
& =\frac{\mathbf{h}_{k+2}^{C}(\tau, 0,1)-\mathbf{h}_{k+1}^{C}(\tau, 1)}{\mathbf{h}_{k+1}^{C}(\tau, 1)+u\left(\mathbf{h}_{k+2}^{C}(\tau, 0,1)-\mathbf{h}_{k+1}^{C}(\tau, 1)\right)} \\
& =\frac{\mathbf{h}_{k+1}^{C}(\tau, 0)}{\mathbf{h}_{k+1}^{C}(\tau, 1)+u \mathbf{h}_{k+1}^{C}(\tau, 0)}=\frac{\mathbf{v}_{k}(1 ; \tau)}{1+u \cdot \mathbf{v}_{k}(1 ; \tau)} \cdot
\end{aligned}
$$


As the following two lemmata show, $-d / d u \mathbf{H}_{k}^{G}(u)$ is weakly ferromagnetic for $u=0$.

Lemma 5.4. For $k \in \mathbb{N}_{0}, t \in \mathbf{G}_{k}^{*}$ and $\#_{k}(t)$ even

$$
\left.\frac{d}{d u} j_{k}^{G}(u ; t)\right|_{u=0}=\left\{\begin{array}{cl}
-\frac{1}{2}, & t=0 \\
0, & \text { otherwise. }
\end{array}\right.
$$

Proof. Setting $s:=\left(t_{k}, \ldots, t_{2}\right) \in \mathbf{G}_{k-1}^{*}$,

$$
\begin{aligned}
\left.\frac{d}{d u} j_{k}^{G}(u ; t)\right|_{u=0} & =-2^{-k} \sum_{\sigma \in \mathbf{G}_{k}} \mathbf{v}_{k}\left(1 ; \sigma_{k}, \ldots, \sigma_{1}\right) \chi_{t}(\sigma) \\
& =-2^{-k} \sum_{\sigma \in \mathbf{G}_{k}} \mathbf{v}_{k}\left(1 ; \sigma_{1}, \ldots, \sigma_{k}\right) \chi_{\left(t_{k}, \ldots, t_{1}\right)}(\sigma) \\
& =-2^{-k} \sum_{\tau \in \mathbf{G}_{k-1}}\left(\mathbf{v}_{k}(1 ;(\tau, 0))+\mathbf{v}_{k}(1 ;(1-\tau, 1))\right) \chi_{s}(\tau) \\
& =-2^{-k} \sum_{\tau \in \mathbf{G}_{k-1}} \chi_{s}(\tau)=-\frac{1}{2} \delta_{s, 0},
\end{aligned}
$$

by (38) and (17). But if $\#_{k}(t)$ is even, then $s=0$ if and only if $t=0$.

Lemma 5.5. For $k \in \mathbb{N}, t \in \mathbf{G}_{k}^{*}$ and $\#_{k}(t)$ odd,

$$
\left.\frac{d}{d u} j_{k}^{G}(u ; t)\right|_{u=0} \geqq 0 \text {. }
$$

Proof. With the same notation as in the proof of the last lemma,

$$
\begin{aligned}
\left.\frac{d}{d u} j_{k}^{G}(u ; t)\right|_{u=0} & =-2^{-k} \sum_{\tau \in \mathbf{G}_{k-1}}\left(\mathbf{v}_{k}(1 ;(\tau, 0))-\mathbf{v}_{k}(1 ;(1-\tau, 1))\right) \chi_{s}(\tau) \\
& =+2^{-k} \sum_{\tau \in \mathbf{G}_{k-1}} \frac{1-\mathbf{v}_{k-1}(1 ; \tau)}{1+\mathbf{v}_{k-1}(1 ; \tau)} \chi_{s}(\tau) \geqq 0,
\end{aligned}
$$

using (39) and Lemma 5.1.

Lemma 5.6. For $k \in \mathbb{N}$ and $t \in \mathbf{G}_{k}^{*} \backslash\{0\}$

$$
\frac{d}{d u} j_{k}^{G}(u ; t) \geqq 0, \quad u \in[0,1] .
$$

Proof. Lemma 5.4 and Lemma 5.5 show that the assertion holds true for $u=0$.

We define $\hat{\mathbf{v}}_{k}: \mathbf{G}_{k} \rightarrow \mathbb{R}$ by $\hat{\mathbf{v}}_{k}:=\frac{1}{2}-\mathbf{v}_{k}(1)$. Then for $t \in \mathbf{G}_{k}^{*} \backslash\{0\}$

$$
\left.\frac{d}{d u} j_{k}^{G}(u ; t)\right|_{u=0}=+\mathscr{F}_{k}\left(\hat{\mathbf{v}}_{k}\right)\left(t_{k}, \ldots, t_{1}\right),
$$

$\hat{\mathbf{v}}_{k}$ is strictly ferromagnetic, and for arbitrary $u \in[0,1]$

$$
\frac{d}{d u} j_{k}^{G}(u ; t)=+\mathscr{F}_{k}\left(\mathrm{~g}_{u}\left(\hat{\mathbf{v}}_{k}\right)\right)\left(t_{k}, \ldots, t_{1}\right), \quad t \in \mathbf{G}_{k}^{*} \backslash\{0\},
$$


with

$$
\mathrm{g}_{u}:\left(-\frac{1}{2}, \frac{1}{2}\right) \rightarrow \mathbb{R}, \quad \mathrm{g}_{u}(x):=\frac{x-\frac{1}{2}}{1-u\left(x-\frac{1}{2}\right)}+\frac{1}{2}
$$

$\mathrm{g}_{u}(0)=\frac{1}{2}\left(1-\frac{1}{1+u / 2}\right) \geqq 0$, and for $n \in \mathbb{N}$

$$
\left.\frac{d^{n}}{d x^{n}} \mathrm{~g}_{u}(x)\right|_{x=0}=(1+u / 2)^{-2} n !\left(\frac{u}{1+u / 2}\right)^{n-1} \geqq 0 \text {. }
$$

Moreover, $g_{u}$ is real-analytic on the interval $(-1 / 2,1 / 2)$ for $u \in[0,1]$.

Thus by Proposition $3.2, \mathrm{~g}_{u}\left(\hat{\mathbf{v}}_{k}\right)$ is strictly ferromagnetic, so that the lemma follows from (50).

Now we have proven the ferromagnetic property for both the grand canonical and the canonical ensemble:

Proposition 5.7. For $k \in \mathbb{N}$ and $t=\left(t_{1}, \ldots, t_{k}\right) \in \mathbf{G}_{k}^{*} \backslash\{0\}$ with $\#_{k}(t)$ even,

$$
j_{k}^{G}(t) \geqq \begin{cases}0, & t_{1}=1 \\ j_{k-1}^{G}\left(t_{2}, \ldots, t_{k}\right), & t_{1}=0 .\end{cases}
$$

Both the canonical and the grand canonical ensembles are weakly ferromagnetic, i.e.,

$$
j_{k}^{C}(t) \geqq 0 \text { and } j_{k}^{G}(t) \geqq 0 \text { for all } t \in \mathbf{G}_{k}^{*} \backslash\{0\} .
$$

Furthermore, for $t \in \mathbf{G}_{k}^{*} \backslash\{0\}$,

$$
j_{k}^{C}(t) \geqq j_{k+1}^{C}(t, 0) \geqq \frac{1}{2} j_{k}^{C}(t) \geqq j_{k+1}^{C}(t, 1) \geqq 0 .
$$

Proof. (51) follows, since by (48) and (49)

using Lemma 5.6.

$$
\begin{aligned}
j_{k}^{G}(t) & =j_{k-1}^{G}\left(t_{2}, \ldots, t_{k}\right)+\int_{0}^{1} \frac{d}{d u} j_{k-1}^{G}(u ; t) d u \\
& \geqq j_{k-1}^{G}\left(t_{2}, \ldots, t_{k}\right),
\end{aligned}
$$

Observe that $j_{k}^{G}(t)=j_{k-1}^{G}\left(t_{2}, \ldots, t_{k}\right)+\int_{0}^{1} \frac{d}{d u} j_{k-1}^{G}(u ; t) d u$ even if $t_{1}=1$, since then $j_{k-1}^{G}\left(t_{2}, \ldots, t_{k}\right)=0$ by Proposition 4.3 .

Observe further that we may assume $k>1$ since there does not exist a $t \in \mathbf{G}_{1}^{*} \backslash\{0\}$ with $\#_{k}(t)$ even. Thus we can apply Lemma 5.6.

The grand canonical ensemble is weakly ferromagnetic as a consequence of (51), and of the vanishing of the $j_{k}^{G}(t)$ for $\#_{k}(t)$ odd (Proposition 4.3).

The canonical ensemble is weakly ferromagnetic by Corollary 5.2, and since

$$
j_{k+1}^{C}(t, 0)=j_{k+1}^{C}(t, 1)+j_{k}^{G}(t)
$$

by Lemma 4.2. By Lemma 4.1

$$
j_{k+1}^{C}(t, 0)+j_{k+1}^{C}(t, 1)=j_{k}^{C}(t),
$$

so that (52) follows. 


\section{Upper Bounds for the Interaction Coefficients}

In this section we derive bounds for $j_{k}^{c}(t)$ depending on the form of $t \in \mathbf{G}_{k}^{*}$.

The principal character $t=(0, \ldots, 0)$ plays a special role, for it is obvious that $j_{k}^{C}(0)<0$ and $\lim _{k \rightarrow \infty} j_{k}^{C}(0)=-\infty$ since $j_{k}^{C}(0)$ is minus the mean value of the function $H_{k}^{C}$.

In Proposition 5.7 we proved that $j_{k}^{C}(t) \geqq 0$ for the non-principal characters $t \in \mathbf{G}_{k}^{*} \backslash\{0\}$. Here we shall derive upper bounds for these canonical interaction coefficients.

One quantity which regulates the size of $\left|j_{k}^{C}(t)\right|$ is the value of the counting function $\#_{k}(t)$ on the group $\mathbf{G}_{k}^{*}$ of characters. It will turn out that, roughly speaking, $j_{k}^{C}(t)$ is typically small if $\#_{k}(t)$ is odd.

For $\#_{k}(t)$ even, $\left|j_{k}^{C}(t)\right|$ is small only if the size $S_{k}(t)=P_{k}^{r}(t)-P_{k}^{l}(t)+1$ of $t \in \mathbf{G}_{k}^{*} \backslash\{0\}$ is large.

Although the coefficients $h_{k}^{c}(a)$ are invariant under the reflection $a \mapsto-a$ (see $(8)), \chi_{t}(-a) \neq \chi_{t}(a)$ in general for the functions $\chi_{t}:=\chi_{t} \circ \operatorname{Id}_{k}$ on $G_{k}$. Thus there are many cancellations in the defining relation of $j_{k}^{C}(t)$.

Definition 6.1. Let $t \in \mathbf{G}_{k}^{*}$. Then $a \in G_{k}$ is called positive (negative) if $\chi_{t}(a)+\chi_{t}(-a)$ $=2(-2)$. Otherwise $a$ is called vanishing.

$t \in \mathbf{G}_{k}^{*}$ is called interlaced if the ordered list of the non-vanishing elements of $G_{k}$ is alternating between positive and negative elements.

To calculate $\chi_{t}(-a)$, we must relate $\operatorname{Id}_{k}(-a)$ to $\operatorname{Id}_{k}(a)$.

Lemma 6.2. Let $t=\left(t_{1}, \ldots, t_{k}\right) \in \mathbf{G}_{k}^{*}$ with $t_{k}=1$. Then $t$ is interlaced.

Proof. Let $a, b \in G_{k}$ be non-vanishing in the sense of Definition $6.1, a<b$ w.r.t. the order on $G_{k}$ induced by the complete residue system $\left\{0, \ldots, 2^{k}-1\right\}$, and let $a+i$ be vanishing for $0<i<b-a$.

Then by Lemma 3.1

$$
\begin{aligned}
& \chi_{t}(a)=\chi_{t}(-a)=(-1)^{\#_{k}(t)} \chi_{t}(a-1) \\
& \chi_{t}(b)=\chi_{t}(-b)=(-1)^{\#_{k}(t)} \chi_{t}(b-1)
\end{aligned}
$$

and

$$
\begin{aligned}
\chi_{t}(a+i) & =-\chi_{t}(-(a+i))=(-1)^{\#_{k}(t)+1} \chi_{t}(a+i-1) \\
& =(-1)^{i \cdot\left(\#_{k}(t)+1\right)} \chi_{t}(a)
\end{aligned}
$$

so that

$$
\chi_{t}(b)=(-1)^{(b-a) \cdot\left(\#_{k}(t)+1\right)+1} \chi_{t}(a) .
$$

If $\#_{k}(t)$ is odd, then the exponent in (54) is odd.

If $\#_{k}(t)$ is even, then by (53) $\chi_{t}(a)=\chi_{t}(a-1)$ and $\chi_{t}(b)=\chi_{t}(b-1)$. This implies that both $a$ and $b$, considered as elements of the residue system, are even numbers, since for $c \in G_{k}$ odd

$$
\operatorname{Id}_{k}(c)=\left(\sigma_{1}, \ldots, \sigma_{k-1}, 1\right) \text { and } \operatorname{Id}_{k}(c-1)=\left(\sigma_{1}, \ldots, \sigma_{k-1}, 0\right)
$$

so that $\chi_{t}(c)=(-1)^{t_{k}} \chi_{t}(c-1)$. Again, the exponent in (54) is odd.

Thus in both cases $\chi_{t}(b)=-\chi_{t}(a)$ proving the interlacing property. 
For $t \in \mathbf{G}_{k}^{*}$ we define the auxiliary coefficients $J_{k}(t)$ by

$$
J_{k}(t):=-2^{-k} \sum_{a \in G_{k}} H_{k}^{C}(2 a) \chi_{t}(a) .
$$

Lemma 6.3. For $t=\left(t_{1}, \ldots, t_{k}\right) \in \mathbf{G}_{k}^{*}$

$$
J_{k+1}(0, t)=j_{k}^{C}(t)
$$

and

$$
J_{k}(t)=0 \quad \text { if } t_{1}=1
$$

If $t_{k}=1$, then

$$
\left|j_{k}^{C}(t)-J_{k}(t)\right| \leqq 2^{-k} \cdot \ln 2 .
$$

Proof. (56) follows readily from the definitions (5), (16) and (55):

$$
\begin{aligned}
J_{k+1}(0, t) & =-2^{-(k+1)} \sum_{a \in G_{k+1}} H_{k+1}^{C}(2 a) \chi_{(0, t)}(a) \\
& =-2^{-(k+1)} \sum_{\sigma \in G_{k+1}} \mathbf{H}_{k+1}^{C}\left(\sigma_{2}, \ldots, \sigma_{k+1}, 0\right) \cdot(-1)^{\sum_{i=2}^{k+1} t_{k-1} \sigma_{t}} \\
& =-2 \cdot 2^{-(k+1)} \sum_{\sigma \in G_{k}} \mathbf{H}_{k}^{C}\left(\sigma_{1}, \ldots, \sigma_{k}\right) \cdot \chi_{t}(\sigma)=j_{k}^{C}(t) .
\end{aligned}
$$

Similarly, $J_{1}(1)=0$ and for $k \geqq 2$ and $t_{1}=1$

$$
J_{k}(t)=-2^{-k} \sum_{\sigma \in G_{k}} \mathbf{H}_{k}^{C}\left(\sigma_{2}, \ldots, \sigma_{k}, 0\right) \cdot(-1)^{\sigma_{1}} \cdot(-1)^{\sum_{i=2}^{k} t_{i} \sigma_{k}}=0
$$

by summation over $\sigma_{1}$.

With regard to $(58)$, we have

$$
\begin{aligned}
j_{k}^{C}(t)-J_{k}(t)= & -2^{-k} \sum_{a \in G_{k}}\left(H_{k}^{c}(a)-H_{k}^{C}(2 a)\right) \chi_{t}(a) \\
& =2^{-k}\left(H_{k}^{C}\left(2^{k-1}\right) \chi_{t}\left(2^{k-1}\right)-u\right) \\
& =2^{-k}\left((-1)^{t_{0}} \cdot \ln 2-u\right)
\end{aligned}
$$

with

$$
u:=\sum_{a \in\left\{0, \ldots, 2^{k^{-1}}\right\}}\left(H_{k}^{C}(a)-H_{k}^{c}(2 a)\right)\left(\chi_{t}(a)+\chi_{t}(-a)\right) .
$$

Now by Lemma 6.2 the character $t$ is interlaced since $t_{k}=1$.

Moreover, $H_{k}^{C}(a)-H_{k}^{C}(2 a)$, or equivalently $h_{k}^{C}(a) / h_{k}^{C}(2 a)$, is strictly increasing for $0 \leqq a \leqq 2^{k-1}$, as we show now. We start with the trivial relation

$$
\frac{h_{k}^{C}(0)}{h_{k}^{C}(0)}=\frac{1}{1}<\frac{h_{k}^{C}\left(2^{k-1}\right)}{h_{k}^{C}(0)}=\frac{2}{1}
$$


between the end points. Then we use the inductive definition (5) of the $h_{k}^{C}$ to prove the general statement, observing that

$$
\frac{n_{1}}{d_{1}}<\frac{n_{1}+n_{2}}{d_{1}+d_{2}}<\frac{n_{2}}{d_{2}}
$$

for $n_{1}, n_{2}, d_{1}, d_{2}>0$ if $n_{1} / d_{1}<n_{2} / d_{2}$.

So $u$ is represented by an alternating sum whose terms are strictly increasing in absolute value. This implies that $u$ is bounded by the terms for $a=0$ resp. for $a=2^{k-1}$ :

$$
0 \leqq(-1)^{t_{0}} \cdot u \leqq 2 \ln 2
$$

proving (58).

Proposition 6.4. For $t \in \mathbf{G}_{k}^{*}$ with $\#_{k}(t)$ odd

$$
0 \leqq j_{k}^{C}(t) \leqq\left(2^{-(k-l)}-2^{-k}\right) \ln 2
$$

with $l:=P_{k}^{l}(t)$.

Proof. Let $r:=P_{k}^{r}(t)$ and $s:=S_{k}(t)=r-l+1$. We define the character $t^{\prime} \in \mathbf{G}_{s}^{*}$ by

$$
t^{\prime}=\left(t_{1}^{\prime}, \ldots, t_{s}^{\prime}\right):=\left(t_{l}, \ldots, t_{r}\right) .
$$

From Lemma 4.1 we deduce that

$$
j_{k}^{C}(t)=2^{-(k-r)} j_{r}^{C}\left(t^{\prime \prime}\right)
$$

with $t^{\prime \prime}:=\left(0, t^{\prime}\right) \in \mathbf{G}_{r}^{*}$. Moreover,

$$
\begin{aligned}
j_{r}^{C}\left(t^{\prime \prime}\right) & \leqq J_{s}\left(t^{\prime}\right)+\sum_{i=s}^{r}\left|j_{i}^{C}\left(0, t^{\prime}\right)-J_{i}\left(0, t^{\prime}\right)\right| \\
& =0+\sum_{i=s}^{r} 2^{-i} \ln 2=\left(2^{-s+1}-2^{-r}\right) \cdot \ln 2 .
\end{aligned}
$$

by (56) and (58).

So $j_{k}^{C}(t) \leqq 2^{-(k-r)}\left(2^{-s+1}-2^{-r}\right) \cdot \ln 2$, proving the estimate.

Proposition 6.5. For $t \in \mathbf{G}_{k}^{*} \backslash\{0\}$ with $\#_{k}(t)$ even

$$
0 \leqq j_{k}^{C}(t) \leqq\left(2^{-s+2}-2^{-r+1}-2^{-(k-l)}+2^{-k}\right) \cdot \ln 2
$$

with $l:=P_{k}^{l}(t), r:=P_{k}^{r}(t)$ and $s:=S_{k}(t)=r-l+1$.

Proof. Again, we define $t^{\prime} \in \mathbf{G}_{s}^{*}$ by

$$
t^{\prime}=\left(t^{\prime}{ }_{1}, \ldots, t_{s}^{\prime}\right):=\left(t_{l}, \ldots, t_{r}\right)
$$

and $t^{\prime \prime}:=\left(0, t^{\prime}\right) \in \mathbf{G}_{r}^{*}$. Using (20),

$$
j_{k}^{C}(t)=j_{r}^{C}\left(t^{\prime \prime}\right)-\sum_{i=r+1}^{k} j_{i}^{C}\left(t^{\prime \prime}, 0, \ldots, 0,1\right) .
$$


Now $\#_{i}\left(t^{\prime \prime}, 0, \ldots, 0,1\right)=\#_{k}(t)+1$ which is odd so that by Proposition 6.4

$$
\begin{aligned}
j_{k}^{C}(t) & \leqq\left|j_{r}^{C}\left(t^{\prime \prime}\right)\right|+\sum_{i=r+1}^{k}\left(2^{-(i-l)}-2^{-i}\right) \ln 2 \\
& =\left|j_{r}^{C}\left(t^{\prime \prime}\right)\right|+\left(2^{-s+1}-2^{-(k-l)}-2^{-r}+2^{-k}\right) \ln 2 .
\end{aligned}
$$

But by Lemma 6.3

$$
j_{r}^{C}\left(t^{\prime \prime}\right) \leqq \sum_{i=s}^{r}\left|j_{i}^{C}\left(0, t^{\prime}\right)-J_{i}\left(0, t^{\prime}\right)\right| \leqq\left(2^{-s+1}-2^{-r}\right) \ln 2,
$$

so that (61) follows.

Remark 6.6. Notice that the estimates in Propositions 6.4 and 6.5 are sharp for $t=(1,0, \ldots, 0) \in \mathbf{G}_{k}^{*}$ and $t=(1,0, \ldots, 0,1) \in \mathbf{G}_{k}^{*}$, respectively.

\section{Asymptotic Translation Invariance}

We know from Sect. 6 that the interaction coefficients $j_{k}^{C}(t)$ are small in absolute value unless the size $S_{k}(t)$ is small.

In this section we show that the interaction coefficients are asymptotically invariant with respect to translations (up to edge effects).

Lemma 7.1. For $a \in\left\{0, \ldots, 2^{k}-1\right\}$ the function

$$
H_{k+1}^{G}(a)-H_{k}^{G}(a)
$$

is monotone increasing, and $0<H_{k+1}^{G}(a)-H_{k}^{G}(a)<\ln 2$.

Proof. By definition (24) of $H_{k}^{G}(a)=\mathbf{H}_{k}^{G} \circ \operatorname{Id}_{k}(a)$

$$
H_{k+1}^{G}(a)-H_{k}^{G}(a)=H_{k+2}^{C}(2 a+1)-H_{k+1}^{C}(2 a+1) .
$$

So the assertion follows from the estimates for $H_{k}^{C}(a)$ given in the proof of Lemma 6.3.

Lemma 7.2. Let $t \in \mathbf{G}_{k+1}^{*} \backslash\{0\}$ with $\#_{k+1}(t)$ even and $r:=P_{k+1}^{r}(t)$. Then

$$
\left|j_{k+1}^{G}\left(t_{1}, \ldots, t_{k+1}\right)-j_{k}^{G}\left(t_{2}, \ldots, t_{k+1}\right)\right|<2^{1-r} \ln 2 .
$$

Proof. By Lemma 3.1 we have $\chi_{t}(-a-1)=\chi_{t}(a)$ since $\#_{k+1}(t)$ is even. Moreover, $H_{k+1}^{G}(-a-1)=H_{k+1}^{G}(a)$. Thus

$$
\begin{aligned}
& j_{k+1}^{G}\left(t_{1}, \ldots, t_{k+1}\right)-j_{k}^{G}\left(t_{2}, \ldots, t_{k+1}\right) \\
= & -2^{-(k+1)} \sum_{\sigma \in \mathbf{G}_{k+1}} \mathbf{H}_{k+1}^{G}(\sigma) \chi_{t}(\sigma)+2^{-k} \sum_{\sigma \in \mathbf{G}_{k}} \mathbf{H}_{k}^{G}(\sigma) \chi_{\left(t_{2}, \ldots, t_{k+1}\right)}(\sigma) \\
= & -2^{-k} \sum_{a \in\left\{0, \ldots, 2^{k}-1\right\}}\left(H_{k+1}^{G}(a)-H_{k}^{G}(a)\right) \chi_{\left(t_{2}, \ldots, t_{k+1}\right)}(a) .
\end{aligned}
$$


We split that sum by writing

with

$$
\begin{gathered}
\sum_{a \in\left\{0, \ldots, 2^{k-1}\right\}}\left(H_{k+1}^{G}(a)-H_{k}^{G}(a)\right) \chi_{\left(t_{2}, \ldots, t_{k+1}\right)}(a) \\
=\sum_{\left(\sigma_{r+1}, \ldots, \sigma_{k+1}\right)}\left(-\sum_{\substack{\left(\sigma_{2}, \ldots, \sigma_{r-1}\right) \\
\sum_{i=2}^{r-1} \sigma_{t_{1}=\text { even }}}} A\left(\left(\sigma_{2}, \ldots, \sigma_{r-1}\right),\left(\sigma_{r+1}, \ldots, \sigma_{k+1}\right)\right)\right. \\
\left.+\sum_{\substack{\left(\sigma_{2}, \ldots, j, \sigma_{r-1}\right) \\
\sum_{l=2}^{r-1} \sigma_{i} t_{l}=\text { odd }}} A\left(\left(\sigma_{2}, \ldots, \sigma_{r-1}\right),\left(\sigma_{r+1}, \ldots, \sigma_{k+1}\right)\right)\right)
\end{gathered}
$$

$$
\begin{aligned}
A\left(\left(\sigma_{2}, \ldots, \sigma_{r-1}\right),\left(\sigma_{r+1}, \ldots, \sigma_{k+1}\right)\right)= & -\mathbf{H}_{k+1}^{G}\left(0, \sigma_{2}, \ldots, \sigma_{r-1}, 0, \sigma_{r+1}, \ldots, \sigma_{k+1}\right) \\
& +\mathbf{H}_{k}^{G}\left(\sigma_{2}, \ldots, \sigma_{r-1}, 0, \sigma_{r+1}, \ldots, \sigma_{k+1}\right) \\
& +\mathbf{H}_{k+1}^{G}\left(0, \sigma_{2}, \ldots, \sigma_{r-1}, 1, \sigma_{r+1}, \ldots, \sigma_{k+1}\right) \\
& -\mathbf{H}_{k}^{G}\left(\sigma_{2}, \ldots, \sigma_{r-1}, 1, \sigma_{r+1}, \ldots, \sigma_{k+1}\right) .
\end{aligned}
$$

By the monotonicity result of Lemma 7.1 both the sum over the even and the sum over the odd terms in (62) is positive and smaller than $\ln 2$. So their difference is smaller than $\ln 2$ in absolute value.

Due to the summation over $\left(\sigma_{r+1}, \ldots, \sigma_{k+1}\right)$ there are $2^{k+1-r}$ such terms in (62), leading to our estimate.

Proposition 7.3. Let $t \in \mathbf{G}_{k}^{*} \backslash\{0\}$ with $\#_{k}(t)$ even. Then

with $l:=P_{k}^{l}(t)$ and $r:=P_{k}^{r}(t)$.

$$
\left|j_{k+1}^{G}(0, t)-j_{k+1}^{G}(t, 0)\right|<\left(2^{-r}+2^{l-(k+1)}\right) \ln 2
$$

Proof. We denote by $t^{\prime}:=\left(t_{k}, \ldots, t_{1}\right)$ the inverse of $t:=\left(t_{1}, \ldots, t_{k}\right)$. Then

$$
\begin{aligned}
\left|j_{k+1}^{G}(0, t)-j_{k+1}^{G}(t, 0)\right| \leqq & \left|j_{k+1}^{G}(0, t)-j_{k}^{G}(t)\right|+\left|j_{k}^{G}(t)-j_{k}^{G}\left(t^{\prime}\right)\right| \\
& +\left|j_{k}^{G}\left(t^{\prime}\right)-j_{k+1}^{G}\left(0, t^{\prime}\right)\right|+\left|j_{k+1}^{G}\left(0, t^{\prime}\right)-j_{k+1}^{G}(t, 0)\right| .
\end{aligned}
$$

We know from Prop. 4.9 that $j_{k}^{G}\left(t^{\prime}\right)=j_{k}^{G}(t)$ and $j_{k+1}^{G}\left(0, t^{\prime}\right)=j_{k}^{G}(t, 0)$. So we get from Lemma 7.2 that

$$
\begin{aligned}
\left|j_{k+1}^{G}(0, t)-j_{k+1}^{G}(t, 0)\right| & \leqq\left|j_{k+1}^{G}(0, t)-j_{k}^{G}(t)\right|+\left|j_{k}^{G}\left(t^{\prime}\right)-j_{k+1}^{G}\left(0, t^{\prime}\right)\right| \\
& \leqq 2^{1-P_{k+1}^{r}(0, t)} \ln 2+2^{1-P_{k+1}^{r}\left(0, t^{\prime}\right)} \ln 2 \\
& =\left(2^{-r}+2^{l-k-1}\right) \ln 2,
\end{aligned}
$$

since $P_{k+1}^{r}(0, t)=P_{k}^{r}(t)+1$ and $P_{k+1}^{r}\left(0, t^{\prime}\right)=P_{k}^{r}\left(t^{\prime}\right)+1=k+2-P_{k}^{l}(t)$.

\section{Decay Properties of the Potential}

Up to now, only finite spin chains of length $k \in \mathbb{N}_{0}$ were treated. The thermodynamic limit $k \rightarrow \infty$ can exist only if the positions of spins $\sigma_{l}, \sigma_{r}$ far apart within the chain (that is, $|r-l|$ large) are not too dependent of each other. 
More precisely, the probability measure on the group $\mathbf{G}_{k} \equiv\left(\mathbb{Z}_{2}\right)^{k}$ for inverse temperature $s$ given by the distribution

$$
\frac{1}{Z_{k}(s)} \exp \left(-s \mathbf{H}_{k}^{c}\right): \mathbf{G}_{k} \rightarrow[0,1]
$$

should not deviate too much from a product measure on $\mathbf{G}_{k}$.

The negative Fourier transform $j_{k}^{C}=-\mathscr{F}_{k}\left(\mathbf{H}_{k}^{C}\right)$ of the canonical energy function plays the role of a potential. In Sect. 7 it was shown that this potential is asymptotically translation invariant. It is well-known (see Ruelle [6], Chap. 2) that the thermodynamic limit exists if the potential decays fast enough.

In this section we will prove such decay properties for the canonical and the grand canonical ensembles.

For $l, r, k \in \mathbb{N}$ with $1 \leqq l<r \leqq k$ let

$$
A_{k}^{C}(l, r):=\sum_{t \in \mathbf{G}_{r-l-1}^{*}} j_{k}^{C}\left(0_{l-1}, 1, t, 1,0_{k-r}\right),
$$

$0_{s} \in \mathbf{G}_{s}$ denoting the identity element of the group.

Similarly, we define

$$
A_{k}^{G}(l, r):=\sum_{t \in \mathbf{G}_{r-l-1}^{*}} j_{k}^{G}\left(0_{l-1}, 1, t, 1,0_{k-r}\right)
$$

for the grand canonical ensemble.

All terms in these sums are positive, as follows from the weak ferromagnetic properties shown in Sect. 5 .

We shall show that the potential decays in the sense that, independent of $k$, $A_{k}^{C}(l, r)$ and $A_{k}^{G}(l, r)$ are small if $r-l$ is large.

We begin with the canonical ensemble.

$$
A_{k}^{C}(l, r)=-2^{-k} \sum_{\sigma \in \mathbf{G}_{k}} \sum_{t \in \mathbf{G}_{r-l-1}^{*}} \mathbf{H}_{k}^{C}(\sigma) \chi_{s}(\sigma)
$$

for $s:=\left(0_{l-1}, 1, t, 1,0_{k-r}\right) \in \mathbf{G}_{k}^{*}$. Thus using $\mathbf{H}_{k}^{C}=\ln \left(\mathbf{h}_{k}^{C}\right)$,

$$
\begin{aligned}
A_{k}^{C}(l, r)= & -2^{-k} \sum_{\sigma^{I} \in \mathbf{G}_{l-1}} \sum_{\sigma^{I I} \in \mathbf{G}_{k-r}} \sum_{\tau^{I}, \tau^{I I} \in \mathbf{G}_{1}} \sum_{\rho \in \mathbf{G}_{r-l-1}} \\
& \sum_{t \in \mathbf{G}^{*}-l-1} \mathbf{H}_{k}^{C}\left(\sigma^{I}, \tau^{I}, \rho, \tau^{I I}, \sigma^{I I}\right) \cdot(-1)^{\tau^{I+\tau^{I I}}} \boldsymbol{\chi}_{t}(\rho) \\
= & -2^{-k+r-l-1} \sum_{\sigma^{I} \in \mathbf{G}_{l-1}} \sum_{\sigma^{I I} \in \mathbf{G}_{k-r}} \\
& \left(\ln \left[\frac{\mathbf{h}_{k}^{C}\left(\sigma^{I}, 0,0_{r+l-1}, 0, \sigma^{I I}\right)}{\mathbf{h}_{k}^{C}\left(\sigma^{I}, 0,0_{r+l-1}, 1, \sigma^{I I}\right)}\right]-\ln \left[\frac{\mathbf{h}_{k}^{C}\left(1-\sigma^{I}, 1,0_{r-l-1}, 0, \sigma^{I I}\right)}{\mathbf{h}_{k}^{C}\left(1-\sigma^{I}, 1,0_{r-l-1}, 1, \sigma^{I I}\right)}\right]\right) \\
= & +\frac{1}{4} 2^{-(k-r)-(l-1)} \sum_{\sigma^{I} \in \mathbf{G}_{l-1}} \sum_{\sigma^{I I} \in \mathbf{G}_{k-r}} \ln \left[\frac{\mathbf{v}_{r-1, k-r}\left(\left(1-\sigma^{I}, 1,0_{r-l-1}\right) ; \sigma^{I I}\right)}{\mathbf{v}_{r-1, k-r}\left(\left(\sigma^{I}, 0,0_{r-l-1}\right) ; \sigma^{I I}\right)}\right]
\end{aligned}
$$


where for $k, l \in \mathbb{N}_{0}, \sigma \in \mathbf{G}_{k}$ and $\tau \in \mathbf{G}_{l}$

$$
\mathbf{v}_{k, l}(\sigma ; \tau):=\frac{\mathbf{h}_{k+l+1}^{C}(\sigma, 0, \tau)}{\mathbf{h}_{k+l+1}^{C}(\sigma, 1, \tau)} .
$$

Lemma 8.1. For $k, l \in \mathbb{N}_{0}, \sigma \in \mathbf{G}_{k}$ and $\tau \in \mathbf{G}_{l}$

$$
\mathbf{v}_{k, l}(\sigma ; \tau)=\frac{\mathbf{r}_{l+1}(1,0)(0, \tau) \cdot \mathbf{v}_{k}(1 ; \sigma)+\mathbf{r}_{l+1}(0,1)(0, \tau)}{\mathbf{r}_{l+1}(1,1)(0, \tau)-\mathbf{r}_{l+1}(0,1)(0, \tau) \cdot \mathbf{v}_{k}(1 ; \sigma)},
$$

with $\mathbf{r}_{l}\left(s_{0}, s_{1}\right)$ and $\mathbf{v}_{k}(u ; \sigma)$ defined in Sect. 4 and Sect. 5, respectively.

Proof. For $u=1$, the interpolating coefficients $\mathbf{v}_{k}(u ; \sigma)$ equal

$$
\mathbf{v}_{k}(1 ; \sigma)=\frac{\mathbf{h}_{k+1}^{C}(\sigma, 0)}{\mathbf{h}_{k+1}^{C}(\sigma, 1)}
$$

We use the relation

$$
\mathbf{h}_{m}^{C}(\rho)=\mathbf{r}_{m+2}(0,1)(0,1, \rho), \quad \rho \in \mathbf{G}_{m},
$$

which is valid for arbitrary $m \in \mathbb{N}_{0}$ since for $m=0$

$$
\mathbf{r}_{2}(0,1)(0,1)=\mathbf{r}_{2}(0,1)(1,0)=1=\mathbf{h}_{0}^{c},
$$

and by induction in $m$. Thus with the abbreviation $\mathbf{r}_{m}(\rho) \equiv \mathbf{r}_{m}(0,1)(\rho)$,

$$
\begin{aligned}
\mathbf{v}_{k, l}(\sigma ; \tau)= & \frac{\mathbf{h}_{k+l+1}^{C}(\sigma, 0, \tau)}{\mathbf{h}_{k+l+1}^{C}(\sigma, 1, \tau)}=\frac{\mathbf{r}_{k+l+3}(0,1)(0,1, \sigma, 0, \tau)}{\mathbf{r}_{k+l+3}(0,1)(0,1, \sigma, 1, \tau)} \\
= & \frac{\mathbf{r}_{l+1}\left(\mathbf{r}_{k+3}(0,1, \sigma, 0), \mathbf{r}_{k+3}(1,0,1-\sigma, 1)\right)(0, \tau)}{\mathbf{r}_{l+1}\left(\mathbf{r}_{k+3}(0,1, \sigma, 1), \mathbf{r}_{k+3}(1,0,1-\sigma, 0)\right)(0, \tau)} \\
= & \frac{\mathbf{r}_{k+3}(0,1, \sigma, 0) \cdot \mathbf{r}_{l+1}(1,0)(0, \tau)+\mathbf{r}_{k+3}(1,0,1-\sigma, 1) \cdot \mathbf{r}_{l+1}(0,1)(0, \tau)}{\mathbf{r}_{k+3}(0,1, \sigma, 1) \cdot \mathbf{r}_{l+1}(1,0)(0, \tau)+\mathbf{r}_{k+3}(1,0,1-\sigma, 0) \cdot \mathbf{r}_{l+1}(0,1)(0, \tau)} \\
= & \frac{\mathbf{r}_{k+3}(0,1, \sigma, 0) \cdot \mathbf{r}_{l+1}(1,0)(0, \tau)+\mathbf{r}_{k+3}(0,1,1-\sigma, 1) \cdot \mathbf{r}_{l+1}(0,1)(0, \tau)}{\mathbf{r}_{k+3}(0,1, \sigma, 1) \cdot \mathbf{r}_{l+1}(1,0)(0, \tau)+\mathbf{r}_{k+3}(0,1,1-\sigma, 0) \cdot \mathbf{r}_{l+1}(0,1)(0, \tau)} \\
= & \frac{\mathbf{h}_{k+1}^{C}(\sigma, 0) \cdot \mathbf{r}_{l+1}(1,0)(0, \tau)+\mathbf{h}_{k+1}^{C}(1-\sigma, 1) \cdot \mathbf{r}_{l+1}(0,1)(0, \tau)}{\mathbf{h}_{k+1}^{C}(\sigma, 1) \cdot \mathbf{r}_{l+1}(1,0)(0, \tau)+\mathbf{h}_{k+1}^{C}(1-\sigma, 0) \cdot \mathbf{r}_{l+1}(0,1)(0, \tau)} \\
= & \frac{\mathbf{h}_{k+1}^{C}(\sigma, 0) \cdot \mathbf{r}_{l+1}(1,0)(0, \tau)+\mathbf{h}_{k+1}^{C}(\sigma, 1) \cdot \mathbf{r}_{l+1}(0,1)(0, \tau)}{\mathbf{h}_{k+1}^{C}(\sigma, 1) \mathbf{r}_{l+1}(1,1)(0, \tau)+\left(\mathbf{h}_{k+1}^{C}(1-\sigma, 0)-\mathbf{h}_{k+1}^{C}(\sigma, 1)\right) \mathbf{r}_{l+1}(0,1)(0, \tau)} \\
= & \frac{\mathbf{r}_{l+1}(1,0)(0, \tau) \cdot \mathbf{v}_{k}(1 ; \sigma)+\mathbf{r}_{l+1}(0,1)(0, \tau)}{\mathbf{r}_{l+1}(1,1)(0, \tau)-\mathbf{r}_{l+1}(0,1)(0, \tau) \cdot \mathbf{v}_{k}(1 ; \sigma)}
\end{aligned}
$$

The third equation follows from Lemma 4.6, the fourth from Lemma 4.4. For the fifth equation we used the relation $\mathbf{r}_{m+2}(0,1, \sigma)=\mathbf{r}_{m+2}(1,0, \sigma)$ which holds since for $\tau=0$ or 1

$$
\begin{aligned}
\mathbf{r}_{m+2}(\tau, 1-\tau, \sigma) & =\mathbf{r}_{m+1}\left(\mathbf{r}_{2}(\tau, 1-\tau), \mathbf{r}_{2}(1-\tau, \tau)\right)(0, \sigma) \\
& =\mathbf{r}_{m+1}(1,1)(0, \sigma) .
\end{aligned}
$$


Again, in the second-to last equation we used Lemma 4.4, and the relations

$$
\mathbf{h}_{k+1}^{C}(\sigma, 1)=\mathbf{h}_{k+1}^{C}(1-\sigma, 1)=\mathbf{h}_{k+1}^{C}(\sigma, 0)+\mathbf{h}_{k+1}^{C}(1-\sigma, 0) .
$$

By Lemma 8.1 the argument of the logarithm in (63) is a quotient of terms of the form

$$
\begin{aligned}
\mathbf{v}_{r-1, k-r}\left(\left(\tilde{\sigma}, \tau^{I}, 0_{r-l-1}\right) ; \sigma^{I I}\right) \\
\quad=\frac{\mathbf{r}_{k-r+1}(1,0)\left(0, \sigma^{I I}\right) \cdot \mathbf{v}_{r-1}\left(1 ;\left(\tilde{\sigma}, \tau^{I}, 0_{r-l-1}\right)\right)+\mathbf{r}_{k-r+1}(0,1)\left(0, \sigma^{I I}\right)}{\mathbf{r}_{k-r+1}(1,1)\left(0, \sigma^{I I}\right)-\mathbf{r}_{k-r+1}(0,1)\left(0, \sigma^{I I}\right) \cdot \mathbf{v}_{r-1}\left(1 ;\left(\tilde{\sigma}, \tau^{I}, 0_{r-l-1}\right)\right)}
\end{aligned}
$$

with $\left(\tilde{\sigma}, \tau^{I}\right)=\left(\sigma^{I}, 0\right)$ or $\left(1-\sigma^{I}, 1\right)$.

But for $m \in \mathbb{N}, \sigma \in \mathbf{G}_{m}, n \in \mathbb{N}_{0}$ and the identity element $0_{n} \in \mathbf{G}_{n}$

$$
\mathbf{v}_{m+n}\left(u ;\left(\sigma, 0_{n}\right)\right)=\frac{\mathbf{v}_{m}(u ; \sigma)}{1+n \cdot \mathbf{v}_{m}(u ; \sigma)} \text { for } u \in[0,1]
$$

since (66) holds for $n=0$, and since by Eq. (39)

$$
\begin{aligned}
\mathbf{v}_{m+n+1}\left(u ;\left(\sigma, 0_{n}, 0\right)\right) & =\frac{\mathbf{v}_{m+n}\left(u ;\left(\sigma, 0_{n}\right)\right)}{1+\mathbf{v}_{m+n}\left(u ;\left(\sigma, 0_{n}\right)\right)} \\
& =\frac{\mathbf{v}_{m}(u ; \sigma)}{\left(1+n \cdot \mathbf{v}_{m}(u ; \sigma)\right)+\mathbf{v}_{m}(u ; \sigma)} \\
& =\frac{\mathbf{v}_{m}(u ; \sigma)}{1+(n+1) \cdot \mathbf{v}_{m}(u ; \sigma)} .
\end{aligned}
$$

Applying (66) to our situation, we obtain

$$
\mathbf{v}_{r-1}\left(1 ;\left(\sigma^{I}, 0,0_{r-l-1}\right)\right)=\frac{\mathbf{v}_{l-1}\left(1 ; \sigma^{I}\right)}{1+(r-l) \mathbf{v}_{l-1}\left(1 ; \sigma^{I}\right)}
$$

and

$$
\begin{aligned}
\mathbf{v}_{r-1}\left(1 ;\left(1-\sigma^{I}, 1,0_{r-l-1}\right)\right) & =\frac{\mathbf{v}_{l}\left(1 ;\left(1-\sigma^{I}, 1\right)\right)}{1+(r-l-1) \mathbf{v}_{l}\left(1 ;\left(1-\sigma^{I}, 1\right)\right)} \\
& =\frac{1}{\left(1+\mathbf{v}_{l-1}\left(1 ; \sigma^{I}\right)\right)+(r-l-1)} \\
& =\frac{1}{r-l+\mathbf{v}_{l-1}\left(1 ; \sigma^{I}\right)},
\end{aligned}
$$

where we used the identity $\mathbf{v}_{l}(u ;(1-\sigma, 1))=\left(1+\mathbf{v}_{l-1}(u ; \sigma)\right)^{-1}$.

Inserting (67) resp. (68) in (65), we get

$$
\begin{aligned}
& \mathbf{v}_{r-1, k-r}\left(\left(\sigma^{I}, 0,0,-l-1\right) ; \sigma^{I I}\right) \\
& \quad=\frac{\mathbf{r}_{k-r+1}(1,0)\left(0, \sigma^{I I}\right) \cdot \mathbf{v}_{l-1}\left(1 ; \sigma^{I}\right)+\mathbf{r}_{k-r+1}(0,1)\left(0, \sigma^{I I}\right) \cdot\left(1+(r-l) \mathbf{v}_{l-1}\left(1 ; \sigma^{I}\right)\right)}{\mathbf{r}_{k-r+1}(1,1)\left(0, \sigma^{I I}\right) \cdot\left(1+(r-l) \mathbf{v}_{l-1}\left(1 ; \sigma^{I}\right)\right)-\mathbf{r}_{k-r+1}(0,1)\left(0, \sigma^{I I}\right) \cdot \mathbf{v}_{l-1}\left(1 ; \sigma^{I}\right)}
\end{aligned}
$$


and

$$
=\frac{\mathbf{v}_{r-1, k-r}\left(\left(1-\sigma^{I}, 1,0_{r-l-1}\right) ; \sigma^{I I}\right)}{\mathbf{r}_{k-r+1}(1,0)\left(0, \sigma^{I I}\right)+\mathbf{r}_{k-r+1}(0,1)\left(0, \sigma^{I I}\right) \cdot\left(r-l+\mathbf{v}_{l-1}\left(1 ; \sigma^{I}\right)\right)} .
$$

For $\quad \sigma^{I I}=0 \quad$ we have $\quad \mathbf{r}_{k-r+1}(1,0)\left(0, \sigma^{I I}\right)=\mathbf{r}_{k-r+1}(1,1)\left(0, \sigma^{I I}\right)=1 \quad$ and $\mathbf{r}_{k-r+1}(0,1)\left(0, \sigma^{I I}\right)=0$ so that the argument of the logarithm in (63) acquires the form

$$
\begin{aligned}
& \frac{\mathbf{v}_{r-1, k-r}\left(\left(1-\sigma^{I}, 1,0_{r-l-1}\right) ; 0\right)}{\mathbf{v}_{r-1, k-r}\left(\left(\sigma^{I}, 0,0_{r-l-1}\right) ; 0\right)} \\
& \quad=\frac{1+(r-l) \cdot \mathbf{v}_{l-1}\left(1 ; \sigma^{I}\right)}{\left(r-l+\mathbf{v}_{l-1}\left(1 ; \sigma^{I}\right)\right) \cdot \mathbf{v}_{l-1}\left(1 ; \sigma^{I}\right)}=\frac{1+\frac{1}{r-l} \mathbf{v}_{l-1}^{-1}\left(1 ; \sigma^{I}\right)}{1+\frac{1}{r-l} \mathbf{v}_{l-1}\left(1 ; \sigma^{I}\right)} \\
& \quad=\left[1+\frac{1}{r-l}\left(\mathbf{v}_{l-1}^{-1}\left(1 ; \sigma^{I}\right)-\mathbf{v}_{l-1}\left(1 ; \sigma^{I}\right)\right)\right] \cdot R_{1}\left(\sigma^{I}\right)
\end{aligned}
$$

with

$$
R_{1}\left(\sigma^{I}\right)=\frac{1+\frac{1}{r-l} \mathbf{v}_{l-1}^{-1}\left(1 ; \sigma^{I}\right)}{1+\frac{1}{r-l} \mathbf{v}_{l-1}^{-1}\left(1 ; \sigma^{I}\right)+\left(\frac{1}{r-l}\right)^{2}\left(1-\mathbf{v}_{l-1}^{2}\left(1 ; \sigma^{I}\right)\right)}
$$

For $\sigma^{I I} \in \mathbf{G}_{k-r} \backslash\{0\}$ we may divide through $\mathbf{r}_{k-r+1}(0,1)\left(0, \sigma^{I I}\right)>0$ so that with $n:=k-r+1$ and $\tilde{\sigma}^{I I}:=\left(0, \sigma^{I I}\right)$

$$
\begin{aligned}
\frac{\mathbf{v}_{r-1, k-r}\left(\left(1-\sigma^{I}, 1,0,-l-1\right) ; \sigma^{I I}\right)}{\mathbf{v}_{r-1, k-r}\left(\left(\sigma^{I}, 0,0_{r-l-1}\right) ; \sigma^{I I}\right)} \\
=\frac{\mathbf{r}_{n}(1,0)\left(\tilde{\sigma}^{I I}\right)+\mathbf{r}_{n}(0,1)\left(\tilde{\sigma}^{I I}\right) \cdot\left(r-l+\mathbf{v}_{l-1}\left(1 ; \sigma^{I}\right)\right)}{\mathbf{r}_{n}(1,0)\left(\tilde{\sigma}^{I I}\right)+\mathbf{r}_{n}(0,1)\left(\tilde{\sigma}^{I I}\right) \cdot\left(r-l+\mathbf{v}_{l-1}^{-1}\left(1 ; \sigma^{I}\right)\right)} \\
\\
\cdot \frac{\mathbf{r}_{n}(1,1)\left(\tilde{\sigma}^{I I}\right) \cdot\left(r-l+\mathbf{v}_{l-1}^{-1}\left(1 ; \sigma^{I}\right)\right)-\mathbf{r}_{n}(0,1)\left(\tilde{\sigma}^{I I}\right)}{\mathbf{r}_{n}(1,1)\left(\tilde{\sigma}^{I I}\right) \cdot\left(r-l+\mathbf{v}_{l-1}\left(1 ; \sigma^{I}\right)\right)-\mathbf{r}_{n}(0,1)\left(\tilde{\sigma}^{I I}\right)} \\
=\frac{1+\frac{1}{r-l}\left(\mathbf{v}_{l-1}\left(1 ; \sigma^{I}\right)+\frac{\mathbf{r}_{n}(1,0)\left(\tilde{\sigma}^{I I}\right)}{\mathbf{r}_{n}(0,1)\left(\tilde{\sigma}^{I I}\right)}\right)}{1+\frac{1}{r-l}\left(\mathbf{v}_{l-1}^{-1}\left(1 ; \sigma^{I}\right)+\frac{\mathbf{r}_{n}(1,0)\left(\tilde{\sigma}^{I I}\right)}{\mathbf{r}_{n}(0,1)\left(\tilde{\sigma}^{I I}\right)}\right)} \\
\\
\quad \frac{1+\frac{1}{r-l}\left(\mathbf{v}_{l-1}^{-1}\left(1 ; \sigma^{I}\right)-\frac{\mathbf{r}_{n}(0,1)\left(\tilde{\sigma}^{I I}\right)}{\mathbf{r}_{n}(1,1)\left(\tilde{\sigma}^{I I}\right)}\right)}{1+\frac{1}{r-l}\left(\mathbf{v}_{l-1}\left(1 ; \sigma^{I}\right)-\frac{\mathbf{r}_{n}(0,1)\left(\tilde{\sigma}^{I I}\right)}{\mathbf{r}_{n}(1,1)\left(\tilde{\sigma}^{I I}\right)}\right)}
\end{aligned}
$$




$$
\begin{aligned}
= & 1+\left(\frac{1}{r-l}\right)^{2}\left(\mathbf{v}_{l-1}^{-1}\left(1 ; \sigma^{I}\right)-\mathbf{v}_{l-1}\left(1 ; \sigma^{I}\right)\right) \\
& \cdot\left(\frac{\mathbf{r}_{n}(1,0)\left(\tilde{\sigma}^{I I}\right)}{\mathbf{r}_{n}(0,1)\left(\tilde{\sigma}^{I I}\right)}+\frac{\mathbf{r}_{n}(0,1)\left(\tilde{\sigma}^{I I}\right)}{\mathbf{r}_{n}(1,1)\left(\tilde{\sigma}^{I I}\right)}\right) \cdot R_{2}^{-1}\left(\sigma^{I} ; \sigma^{I I}\right)
\end{aligned}
$$

with

$$
\begin{aligned}
R_{2}\left(\sigma^{I} ; \sigma^{I I}\right)= \\
=1+\frac{1}{r-l}\left(\mathbf{v}_{l-1}^{-1}\left(1 ; \sigma^{I}\right)+\mathbf{v}_{l-1}\left(1 ; \sigma^{I}\right)+\frac{\mathbf{r}_{n}(1,0)\left(\tilde{\sigma}^{I I}\right)}{\mathbf{r}_{n}(0,1)\left(\tilde{\sigma}^{I I}\right)}-\frac{\mathbf{r}_{n}(0,1)\left(\tilde{\sigma}^{I I}\right)}{\mathbf{r}_{n}(1,1)\left(\tilde{\sigma}^{I I}\right)}\right) \\
\quad+\left(\frac{1}{r-l}\right)^{2}\left(1-\frac{\mathbf{r}_{n}(1,0)\left(\tilde{\sigma}^{I I}\right)}{\mathbf{r}_{n}(1,1)\left(\tilde{\sigma}^{I I}\right)}+\mathbf{v}_{l-1}\left(1 ; \sigma^{I}\right) \cdot \frac{\mathbf{r}_{n}(1,0)\left(\tilde{\sigma}^{I I}\right)}{\mathbf{r}_{n}(0,1)\left(\tilde{\sigma}^{I I}\right)}\right. \\
\left.\quad+\mathbf{v}_{l-1}^{-1}\left(1 ; \sigma^{I}\right) \cdot \frac{\mathbf{r}_{n}(0,1)\left(\tilde{\sigma}^{I I}\right)}{\mathbf{r}_{n}(1,1)\left(\tilde{\sigma}^{I I}\right)}\right) \\
=1+\frac{1}{r-l}\left(\mathbf{v}_{l-1}^{-1}\left(1 ; \sigma^{I}\right)+\mathbf{v}_{l-1}\left(1 ; \sigma^{I}\right)-1+\frac{\mathbf{r}_{n}(1,0)\left(\tilde{\sigma}^{I I}\right)}{\mathbf{r}_{n}(0,1)\left(\tilde{\sigma}^{I I}\right)}+\frac{\mathbf{r}_{n}(1,0)\left(\tilde{\sigma}^{I I}\right)}{\mathbf{r}_{n}(1,1)\left(\tilde{\sigma}^{I I}\right)}\right) \\
\quad+\left(\frac{1}{r-l}\right)^{2}\left(\frac{\mathbf{r}_{n}(0,1)\left(\tilde{\sigma}^{I I}\right)}{\mathbf{r}_{n}(1,1)\left(\tilde{\sigma}^{I I}\right)}+\mathbf{v}_{l-1}\left(1 ; \sigma^{I}\right) \cdot \frac{\mathbf{r}_{n}(1,0)\left(\tilde{\sigma}^{I I}\right)}{\mathbf{r}_{n}(0,1)\left(\tilde{\sigma}^{I I}\right)}\right. \\
\left.\quad+\mathbf{v}_{l-1}^{-1}\left(1 ; \sigma^{I}\right) \cdot \frac{\mathbf{r}_{n}(0,1)\left(\tilde{\sigma}^{I I}\right)}{\mathbf{r}_{n}(1,1)\left(\tilde{\sigma}^{I I}\right)}\right),
\end{aligned}
$$

since by Lemma $4.4 \mathbf{r}_{n}(0,1)\left(\tilde{\sigma}^{I I}\right)=\mathbf{r}_{n}(1,1)\left(\tilde{\sigma}^{I I}\right)-\mathbf{r}_{n}(1,0)\left(\tilde{\sigma}^{I I}\right)$.

Now we are ready to estimate $A_{k}^{C}(l, r)$ from above.

We observe that $0<R_{1}\left(\sigma^{I}\right)<1$, since $0<\mathbf{v}_{l-1}\left(1 ; \sigma^{I}\right)<1$ so that the denominator in (70) is larger than the numerator.

On the other hand, $R_{2}\left(\sigma^{I} ; \sigma^{I I}\right)>1$, since trivially

$$
\frac{1}{\mathbf{v}_{l-1}\left(1 ; \sigma^{I}\right)}+\mathbf{v}_{l-1}\left(1 ; \sigma^{I}\right)-1>1
$$

and since the other terms in (72) have positive sign.

Furthermore, $R_{2}\left(\sigma^{I} ; \sigma^{I I}\right)$ divides a positive term in (71).

Therefore, by (69),

$$
\begin{aligned}
& \ln \left[\frac{\mathbf{v}_{r-1, k-r}\left(\left(1-\sigma^{I}, 1,0_{r-l-1}\right) ; 0\right)}{\mathbf{v}_{r-1, k-r}\left(\left(\sigma^{I}, 0,0_{r-l-1}\right) ; 0\right)}\right] \\
& =\ln \left[1+\frac{1}{r-l}\left(\mathbf{v}_{l-1}^{-1}\left(1 ; \sigma^{I}\right)-\mathbf{v}_{l-1}\left(1 ; \sigma^{I}\right)\right)\right]+\ln \left[R_{1}\left(\sigma^{I}\right)\right] \\
& <\frac{1}{r-l}\left(\mathbf{v}_{l-1}^{-1}\left(1 ; \sigma^{I}\right)-\mathbf{v}_{l-1}\left(1 ; \sigma^{I}\right)\right),
\end{aligned}
$$

using the inequality $\ln (1+x) \leqq x$ valid for $x>-1$. 
Similarly, for $\sigma^{I I} \in \mathbf{G}_{k-r} \backslash\{0\}$ by (71)

$$
\begin{aligned}
\ln & {\left[\frac{\mathbf{v}_{r-1, k-r}\left(\left(1-\sigma^{I}, 1,0_{r-l-1}\right) ; \sigma^{I I}\right)}{\mathbf{v}_{r-1, k-r}\left(\left(\sigma^{I}, 0,0_{r-l-1}\right) ; \sigma^{I I}\right)}\right] } \\
< & \left(\frac{1}{r-l}\right)^{2}\left(\mathbf{v}_{l-1}^{-1}\left(1 ; \sigma^{I}\right)-\mathbf{v}_{l-1}\left(1 ; \sigma^{I}\right)\right) \\
& \cdot\left(\frac{\mathbf{r}_{k-r+1}(1,0)\left(0, \sigma^{I I}\right)}{\mathbf{r}_{k-r+1}(0,1)\left(0, \sigma^{I I}\right)}+\frac{\mathbf{r}_{k-r+1}(0,1)\left(0, \sigma^{I I}\right)}{\mathbf{r}_{k-r+1}(1,1)\left(0, \sigma^{I I}\right)}\right) .
\end{aligned}
$$

By substitution of (73) and (74) in (63) we obtain

$$
A_{k}^{C}(l, r)<\frac{1}{4} B_{1}\left(\left(\frac{1}{r-l}\right)^{2} B_{2}+\frac{2^{-(k-r)}}{r-l}\right)
$$

with

$$
B_{1}:=2^{-(l-1)} \sum_{\sigma^{I} \in \mathrm{G}_{l-1}}\left(\mathbf{v}_{l-1}^{-1}\left(1 ; \sigma^{I}\right)-\mathbf{v}_{l-1}\left(1 ; \sigma^{I}\right)\right)
$$

and

$$
B_{2}:=2^{-(k-r)} \sum_{\sigma^{I I} \in \mathbf{G}_{k-r} \backslash\{0\}}\left(\frac{\mathbf{r}_{k-r+1}(1,0)\left(0, \sigma^{I I}\right)}{\mathbf{r}_{k-r+1}(0,1)\left(0, \sigma^{I I}\right)}+\frac{\mathbf{r}_{k-r+1}(0,1)\left(0, \sigma^{I I}\right)}{\mathbf{r}_{k-r+1}(1,1)\left(0, \sigma^{I I}\right)}\right) .
$$

\section{Lemma 8.2.}

$$
B_{1}=2-2^{-l} \text {. }
$$

Proof. For $l=1$ we have $\mathbf{v}_{0}(1 ; 0)=1 / 2$ so that $B_{1}=\frac{3}{2}$. For $l>1$ the second term in (76) gives

$$
\sum_{\sigma \in \mathbf{G}_{l-1}} \mathbf{v}_{l-1}(1 ; \sigma)=\sum_{\sigma^{\prime} \in \mathbf{G}_{l-2}}\left(\mathbf{v}_{l-1}\left(1 ;\left(\sigma^{\prime}, 0\right)\right)+\mathbf{v}_{l-1}\left(1 ;\left(\sigma^{\prime}, 1\right)\right)\right)=2^{l-2}
$$

by Eq. (39).

The first term is evaluated by recursion.

$$
\begin{aligned}
\sum_{\sigma \in \mathbf{G}_{l-1}} \frac{1}{\mathbf{v}_{l-1}(1 ; \sigma)} & =\sum_{\sigma^{\prime} \in \mathbf{G}_{l-2}}\left(\mathbf{v}_{l-1}^{-1}\left(1 ;\left(\sigma^{\prime}, 0\right)\right)+\mathbf{v}_{l-1}^{-1}\left(1 ;\left(\sigma^{\prime}, 1\right)\right)\right) \\
& =\sum_{\sigma^{\prime} \in \mathbf{G}_{l-2}}\left(1+\mathbf{v}_{l-2}\left(1 ; \sigma^{\prime}\right)\right) \cdot\left(\mathbf{v}_{l-2}^{-1}\left(1 ; \sigma^{\prime}\right)+1\right) \\
& =2 \cdot 2^{l-2}+\sum_{\sigma^{\prime} \in \mathbf{G}_{l-2}}\left(\mathbf{v}_{l-2}\left(1 ; \sigma^{\prime}\right)+\mathbf{v}_{l-2}^{-1}\left(1 ; \sigma^{\prime}\right)\right) \\
& =2^{l-1}+2^{l-3}+\sum_{\sigma^{\prime} \in \mathbf{G}_{l-2}} \mathbf{v}_{l-2}^{-1}\left(1 ; \sigma^{\prime}\right) \\
& =\frac{1}{\mathbf{v}_{0}(1 ; 0)}+\sum_{s=1}^{l-1}\left(2^{l-s}+2^{l-s-2}\right) \\
& =2+\left(2^{l}-2\right)+\left(2^{l-2}-\frac{1}{2}\right)=5 \cdot 2^{l-2}-\frac{1}{2}
\end{aligned}
$$


Thus $B_{1}=2^{-(l-1)}\left(2^{l}-\frac{1}{2}\right)$.

\section{Lemma 8.3.}

$$
B_{2}=2-(4+k-r) 2^{-(k+1-r)} .
$$

Proof. For $r=k$ one has $B_{2}=0$. Thus we assume $r<k$.

$$
\begin{aligned}
\sum_{\sigma \in \mathbf{G}_{k-r}\{0\}} \frac{\mathbf{r}_{k-r+1}(0,1)(0, \sigma)}{\mathbf{r}_{k-r+1}(1,1)(0, \sigma)} & =\sum_{s=1}^{k} \sum_{\sigma^{\prime} \in \mathbf{G}_{s-1}} \frac{\mathbf{r}_{k-r+1}(0,1)\left(0, \sigma^{\prime}, 1,0_{k-r-s}\right)}{\mathbf{r}_{k-r+1}(1,1)\left(0, \sigma^{\prime}, 1,0_{k-r-s}\right)} \\
& =\sum_{s=1}^{k} \sum_{\sigma^{\prime} \in \mathbf{G}_{s-1}} \frac{\mathbf{r}_{s+1}(0,1)\left(0, \sigma^{\prime}, 1\right)}{\mathbf{r}_{s+1}(1,1)\left(0, \sigma^{\prime}, 1\right)} .
\end{aligned}
$$

But by Lemmas 4.4, 4.5 and 4.7

$$
\begin{aligned}
& \sum_{\sigma \in \mathbf{G}_{s-1}} \frac{\mathbf{r}_{s+1}(0,1)(0, \sigma, 1)}{\mathbf{r}_{s+1}(1,1)(0, \sigma, 1)} \\
= & \sum_{\sigma \in \mathbf{G}_{s-1}} \frac{\mathbf{r}_{s+1}(0, \sigma, 1)}{\mathbf{r}_{s+1}(0, \sigma, 1)+\mathbf{r}_{s+1}(1, \sigma, 1)} \\
= & \sum_{\sigma \in \mathbf{G}_{s-1}} \frac{\mathbf{r}_{s+1}\left(0,1, \sigma_{s-1}, \ldots, \sigma_{1}\right)}{\mathbf{r}_{s+1}\left(0,1, \sigma_{s-1}, \ldots, \sigma_{1}\right)+\mathbf{r}_{s+1}\left(1,0,1-\sigma_{s-1}, \ldots, 1-\sigma_{1}\right)} \\
= & \sum_{\tau \in \mathbf{G}_{s-1}} \frac{\mathbf{h}_{s-1}^{C}(\tau)}{\mathbf{h}_{s-1}^{C}(\tau)+\mathbf{h}_{s-1}^{C}(1-\tau)}=\sum_{\tau \in \mathbf{G}_{s-1}} \frac{\mathbf{h}_{s}^{C}(\tau, 0)}{\mathbf{h}_{s}^{C}(\tau, 1)} \\
= & \sum_{\tau \in \mathbf{G}_{s-1}} \mathbf{v}_{s-1}(1 ; \tau)=2^{s-2}
\end{aligned}
$$

as in Lemma 8.2. Thus

$$
\sum_{\sigma \in \mathbf{G}_{k-r} \backslash\{0\}} \frac{\mathbf{r}_{k-r+1}(0,1)(0, \sigma)}{\mathbf{r}_{k-r+1}(1,1)(0, \sigma)}=\sum_{s=1}^{k-r} 2^{s-2}=2^{k-r-1}-\frac{1}{2} .
$$

Now we evaluate the first term in (77).

$$
\begin{aligned}
& \sum_{\left.\sigma \in \mathbf{G}_{k-r} \backslash 0\right\}} \frac{\mathbf{r}_{k-r+1}(1,0)(0, \sigma)}{\mathbf{r}_{k-r+1}(0,1)(0, \sigma)} \\
= & \sum_{s=1}^{k-r} \sum_{\sigma^{\prime} \in \mathbf{G}_{s-1}} \frac{\mathbf{r}_{s+1}(1,0)\left(0, \sigma^{\prime}, 1\right)}{\mathbf{r}_{s+1}(0,1)\left(0, \sigma^{\prime}, 1\right)} \\
= & \sum_{s=1}^{k-r} \sum_{\sigma^{\prime} \in \mathbf{G}_{s-1}}\left(\frac{\mathbf{r}_{s+1}(1,1)\left(0, \sigma^{\prime}, 1\right)}{\mathbf{r}_{s+1}(0,1)\left(0, \sigma^{\prime}, 1\right)}-1\right) \\
= & \sum_{s=1}^{k-r} \sum_{\tau \in \mathbf{G}_{s-1}}\left(\mathbf{v}_{s-1}^{-1}(1 ; \tau)-1\right)=\sum_{s=1}^{k-r}\left(5 \cdot 2^{s-2}-\frac{1}{2}-2^{s-1}\right) \\
= & 3\left(2^{k-r-1}-\frac{1}{2}\right)-\frac{k-r}{2},
\end{aligned}
$$


using the same transformations as in (79) for the third equation. The fourth equation follows from (78). Thus

$$
B_{2}=2^{-(k-r)}\left(2^{k-r+1}-\frac{4+k-r}{2}\right),
$$

finishing the proof of Lemma 8.3.

Now we can evaluate the decay rates of the potentials for the canonical and the grand canonical ensemble:

Proposition 8.4. For $l, r, k \in \mathbb{N}$ with $1 \leqq l<r \leqq k$

$$
A_{k}^{C}(l, r) \leqq\left(\frac{1}{r-l}\right)^{2}+\frac{2^{-(k-r+1)}}{r-l}
$$

and

$$
A_{k}^{G}(l, r) \leqq\left(\frac{1}{r-l}\right)^{2} .
$$

Proof. The estimate for the canonical ensemble follows directly from (75), using Lemmas 8.2 and 8.3.

We follow the above lines to prove the estimate for the grand canonical ensemble. Setting $s:=\left(0_{l-1}, 1, t, 1,0_{k-r}\right) \in \mathbf{G}_{k}^{*}$,

$$
\begin{aligned}
& A_{k}^{G}(l, r)=\sum_{t \in \mathbf{G}_{k-l-1}^{*}} j_{k}^{G}(s)=-2^{-k} \sum_{t \in \mathbf{G}_{r-l-1}^{*}} \sum_{\sigma \in \mathbf{G}_{k}} \mathbf{H}_{k}^{G}(\sigma) \chi_{s}(\sigma) \\
&=-2^{-k} \sum_{t \in \mathbf{G}_{r-l-1}^{*}} \sum_{\sigma \in \mathbf{G}_{r}} \mathbf{H}_{k+1}^{C}(\sigma, 1) \chi_{s}(\sigma) \\
&=\frac{1}{4} 2^{-(l-1)-(k-r)} \sum_{\sigma^{I} \in \mathbf{G}_{l-1}} \sum_{\sigma^{I I} \in \mathbf{G}_{k-r}} \ln \left[\frac{\mathbf{v}_{r-1, k-r+1}\left(\left(1-\sigma^{I}, 1,0_{r-l-1}\right) ;\left(\sigma^{I I}, 1\right)\right)}{\mathbf{v}_{r-1, k-r+1}\left(\left(\sigma^{I}, 0,0_{r-l-1}\right) ;\left(\sigma^{I I}, 1\right)\right)}\right] \\
&= \frac{1}{4} 2^{-(l-1)-(k-r)} \sum_{\sigma^{I} \in \mathbf{G}_{l-1}} \sum_{\sigma^{I I} \in \mathbf{G}_{k-r}} \ln \left[1+\left(\frac{1}{r-l}\right)^{2}\left(\mathbf{v}_{l-1}^{-1}\left(1 ; \sigma^{I}\right)-\mathbf{v}_{l-1}\left(1 ; \sigma^{I}\right)\right)\right. \\
&\left.\cdot\left(\frac{\mathbf{r}_{k-r+2}(1,0)\left(0, \sigma^{I I}, 1\right)}{\mathbf{r}_{k-r+2}(0,1)\left(0, \sigma^{I I}, 1\right)}+\frac{\mathbf{r}_{k-r+2}(0,1)\left(0, \sigma^{I I}, 1\right)}{\mathbf{r}_{k-r+2}(1,1)\left(0, \sigma^{I I}, 1\right)}\right) \cdot R_{2}^{-1}\left(\sigma^{I} ;\left(\sigma^{I I}, 1\right)\right)\right] \\
&< \frac{1}{4} 2^{-(k-r)} \frac{B_{1}}{(r-l)^{2}} \sum_{\sigma^{I I} \in \mathbf{G}_{k-r}}\left(\frac{\mathbf{r}_{k-r+2}(1,0)\left(0, \sigma^{I I}, 1\right)}{\mathbf{r}_{k-r+2}(0,1)\left(0, \sigma^{I I}, 1\right)}+\frac{\mathbf{r}_{k-r+2}(0,1)\left(0, \sigma^{I I}, 1\right)}{\mathbf{r}_{k-r+2}(1,1)\left(0, \sigma^{I I}, 1\right)}\right) \\
&=\left.\left(\frac{1}{r-l}\right)^{2} \frac{B_{2}}{2} \cdot \frac{2^{k-r+1}-\frac{1}{2}}{2^{k-r+1}}<\frac{1}{r-l}\right)^{2},
\end{aligned}
$$

using (71), Lemma 8.2, and Eqs. (79) and (80) in Lemma 8.3.

\section{A. Numerical Calculations}

Figure 1 shows the canonical energy function $H_{k}^{C}(a)$ depending on $0 \leqq a<2^{k}$ for $k=10$. 


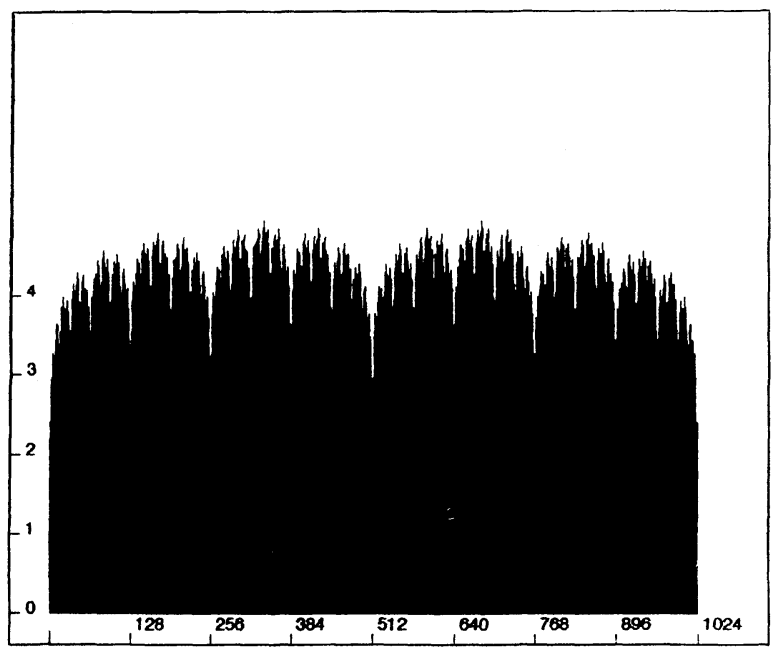

Fig. 1. The graph of the canonical energy function $H_{k}^{C}$ for $k=10$

The basic symmetry is the invariance under the map $a \mapsto 2^{10}-a$.

The fractal (that is, roughly self-similar) structure of the picture is due to the smallness of the difference between $H_{k}^{C}(a)$ and $H_{k}^{C}(2 a)$ for $0 \leqq a<2^{k-1}$.

In fact, as shown in Lemma $6.3, H_{k}^{C}(a)-H_{k}^{C}(2 a)$ is strictly increasing in $a$. That function is shown in Fig. 2.

The canonical interaction coefficients $j_{k}^{C}(t)$ are larger than zero for $t \neq 0$, but as analysed in Propositions 6.4 and 6.5, their absolute values are typically small if $\#_{k}(t)$ is odd.

In Fig. 3 we show the function $j_{k}^{C}(t)$ in a double logarithmic scale.

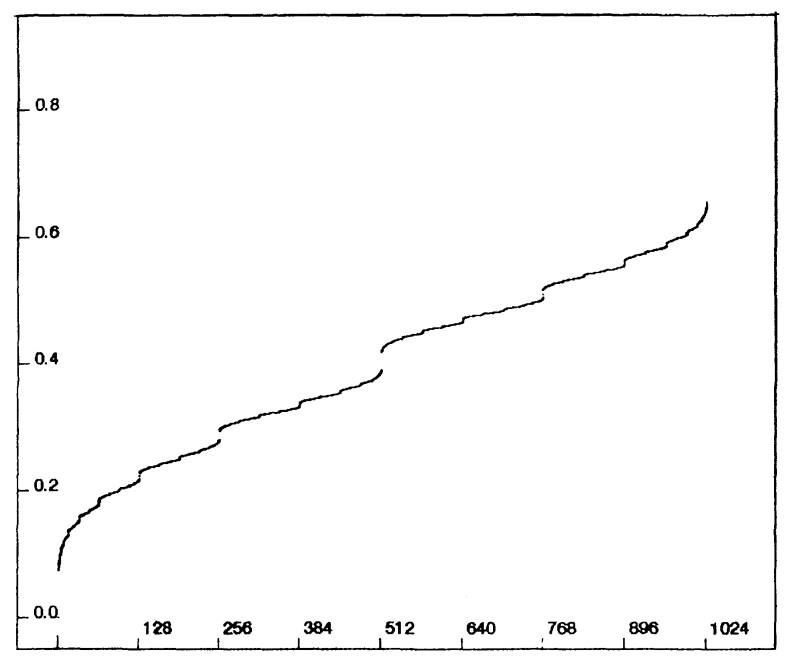

Fig. 2. The function $H_{k}^{c}(a)-H_{k}^{c}(2 a)$ for $k=11$ 


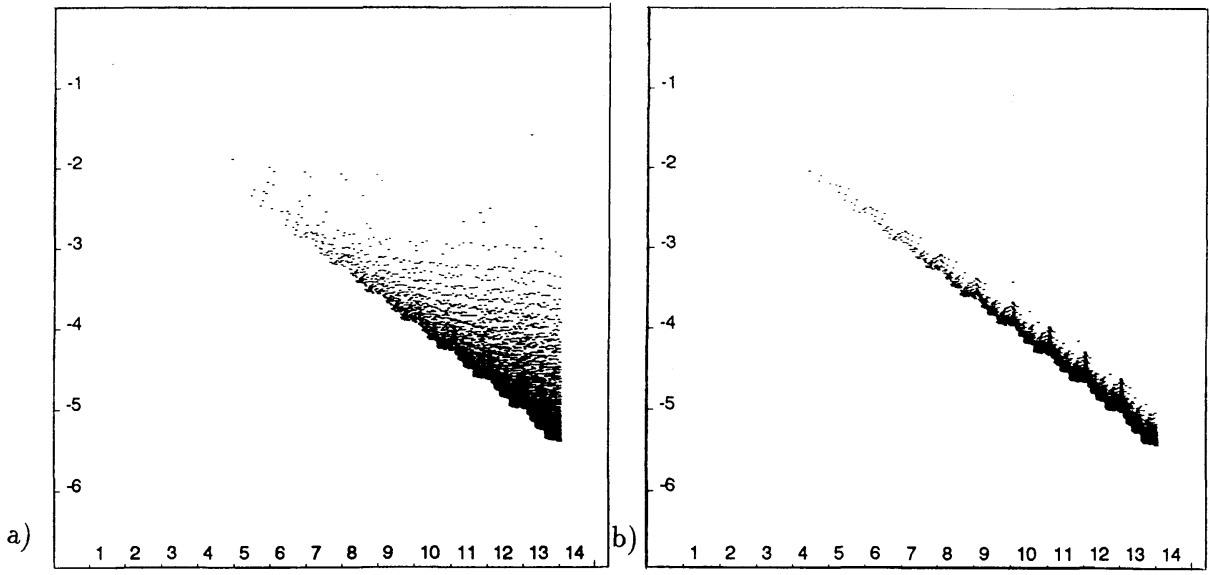

Fig. 3. The logarithms $\log _{10}\left(j_{k}^{C}(t)\right.$ ) (for $t \in \mathbf{G}_{k}^{*} \backslash\{0\}$ ) of the canonical interaction coefficients for $k=14$ and a) $\#_{k}(t)$ even, b) $\#_{k}(t)$ odd

More precisely, we show the graph of $j_{k}^{c} \circ \operatorname{Id}_{k}$ with $\operatorname{Id}_{k}:\left\{0, \ldots, 2^{k}-1\right\} \rightarrow \mathbf{G}_{k}^{*}$ defined similarly to (15). In other words, the abscissa gives the value of $\log _{2}\left(\sum_{l=1}^{k} 2^{k+1-l} t_{l}\right)$ and the ordinate equals $\log _{10}\left(j_{k}^{C}(t)\right)$.

For $\#_{k}(t)$ even (shown in $\left.3 a\right)$ ) the leading coefficients with values of about $\log _{10}\left(j_{k}^{C}(t)\right) \approx-0.9$ are the interaction coefficients for adjacent pairs of spins.

The grand canonical interaction coefficients $j_{k}^{G}(t)$ are shown in Fig. 4 . Only the coefficients with $\#_{k}(t)$ even are taken into account, since the others are zero, as shown in Proposition 4.3.

The symmetry $j_{k}^{G}\left(t_{1}, \ldots, t_{k}\right)=j_{k}^{G}\left(t_{k}, \ldots, t_{1}\right)$ shown in Proposition 4.9 is visible in the picture.

Again, the leading coefficients with values of about $\log _{10}\left(j_{k}^{G}(t)\right) \approx-0.9$ are the interaction coefficients for adjacent pairs of spins.

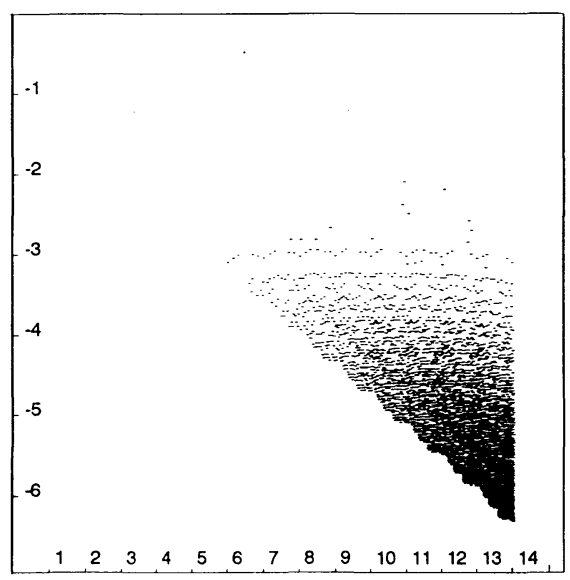

Fig. 4. The logarithms $\log _{10}\left(j_{k}^{G}(t)\right)$ (for $\left.t \in \mathbf{G}_{k}^{*} \backslash\{0\}\right)$ of the grand canonical interaction coefficients for $k=14$ and $\#_{k}(t)$ even 
That is, $\left(t_{l}, \ldots, t_{r}\right)=(1,1)$ for $l:=P_{k}^{l}(t)$ and $r:=P_{k}^{r}(t)$.

The next row with $\log _{10}\left(j_{k}^{G}(t)\right) \approx-1.2$ corresponds to second-nearest neighbour interactions, i.e. $\left(t_{l}, \ldots, t_{r}\right)=(1,0,1)$.

For $\left(t_{l}, \ldots, t_{r}\right)=(1,0,0,1)$ we have $\log _{10}\left(j_{k}^{G}(t)\right) \approx-1.5$, that row being followed by the coefficients with $\left(t_{l}, \ldots, t_{r}\right)=(1,0,0,0,1)$ and those with $\left(t_{l}, \ldots, t_{r}\right)=(1,1,1,1)$.

As one can see by comparing Fig. 3a) and Fig. 4, boundary effects are less pronounced for the grand canonical ensemble than for the canonical ensemble.

Acknowledgement. I want to thank Professor Ruedi Seiler for supporting this work.

\section{References}

1. Apostol, T.M.: Introduction to Analytic Number Theory. Undergraduate Texts in Mathematics. New York: Springer 1976

2. Ginibre, J.: Correlations in Ising Ferromagnets. In: Cargèse Lectures in Physics, Vol. 4. Kastler, D. ed., Gordon and Breach, London 1970

3. Newman, Ch.: Gaussian Correlation Inequalities for Ferromagnets. Z. Wahrscheinlichkeitstheorie verw. Gebiete 33, 75-93 (1975)

4. Pólya, G.: Collected Papers, Vol. II: Locations of Zeros (Boas, R.P. ed.). Cambridge: M.I.T. Press 1974

5. Ruelle, D.: Is Our Mathematics Natural? The Case of Equilibrium Statistical Mechanics. Bull. AMS 19, 259-268 (1988)

6. Ruelle, D.: Statistical Mechanics: Rigorous Results. Addison-Wesley, Redwood City 1989

7. Ruelle, D.: Some Remarks on the Location of Zeroes of the Partition Function for Lattice Systems. Commun. math. Phys. 31, 265-277 (1973)

Communicated by J.P. Eckmann 
